\title{
MAPK mutations and cigarette smoke promote the pathogenesis of pulmonary Langerhans cell histiocytosis
}

\author{
Huan Liu, ${ }^{1}$ Andrew R. Osterburg, ${ }^{1}$ Jennifer Flury, ${ }^{1}$ Zulma Swank, ${ }^{1}$ Dennis W. McGraw, ${ }^{1,2}$ \\ Nishant Gupta, ${ }^{1,2}$ Kathryn A. Wikenheiser-Brokamp, ${ }^{3,4,5}$ Ashish Kumar, ${ }^{6}$ Abdellatif Tazi, ${ }^{7}$ \\ Yoshikazu Inoue, ${ }^{8}$ Masaki Hirose, ${ }^{8}$ Francis X. McCormack, ${ }^{1,2}$ and Michael T. Borchers ${ }^{1,2}$ \\ 'Division of Pulmonary, Critical Care and Sleep Medicine, Department of Internal Medicine, University of Cincinnati, \\ Cincinnati, Ohio, USA. ${ }^{2}$ Cincinnati Veterans Affairs Medical Center, Cincinnati, Ohio, USA. ${ }^{3}$ Division of Pathology and \\ Laboratory Medicine and ${ }^{4}$ Perinatal Institute, Division of Pulmonary Biology, Cincinnati Children's Hospital Medical \\ Center, Cincinnati, Ohio, USA. ${ }^{5}$ Department of Pathology and Laboratory Medicine, University of Cincinnati, Cincinnati, \\ Ohio, USA. ${ }^{6}$ Division of Bone Marrow Transplantation and Immune Deficiency, Cincinnati Children's Hospital Medical \\ Center, Cincinnati, Ohio, USA. ${ }^{7}$ INSERM UMR-S 976, University Paris-Diderot, Sorbonne Paris Cité, Paris, France. ${ }^{8}$ National \\ Hospital Organization Kinki-Chuo Chest Medical Center, Osaka, Japan.
}

Pulmonary Langerhans cell histiocytosis (PLCH) is a rare smoking-related lung disease characterized by dendritic cell (DC) accumulation, bronchiolocentric nodule formation, and cystic lung remodeling. Approximately $50 \%$ of patients with PLCH harbor somatic BRAF-V600E mutations in cells of the myeloid/monocyte lineage. However, the rarity of the disease and lack of animal models have impeded the study of PLCH pathogenesis. Here, we establish a cigarette smoke-exposed (CS-exposed) BRAF-V600E-mutant mouse model that recapitulates many hallmark characteristics of PLCH. We show that CD11c-targeted expression of BRAF-V600E increases DC responsiveness to stimuli, including the chemokine CCL20, and that mutant cell accumulation in the lungs of CS-exposed mice is due to both increased cellular viability and enhanced recruitment. Moreover, we report that the chemokine CCL7 is secreted from DCs and human peripheral blood monocytes in a BRAF-V600E-dependent manner, suggesting a possible mechanism for recruitment of cells known to dominate PLCH lesions. Inflammatory lesions and airspace dilation in BRAF-V600E mice in response to CS are attenuated by transitioning animals to filtered air and treatment with a BRAF-V600E inhibitor, PLX4720. Collectively, this model provides mechanistic insights into the role of myelomonocytic cells and the BRAF-V600E mutation and CS exposure in PLCH pathogenesis and provides a platform to develop biomarkers and therapeutic targets.

Conflict of interest: The authors have declared that no conflict of interest exists.

Copyright: () 2020, American Society for Clinical Investigation.

Submitted: July 24, 2019

Accepted: January 15, 2020

Published: February 27, 2020

Reference information: /CI Insight. 2020;5(4):e132048.

https://doi.org/10.1172/jici.

insight.132048.

\section{Introduction}

Pulmonary Langerhans cell histiocytosis (PLCH) is a rare smoking-related interstitial lung disease characterized by bronchiolocentric, Langerin ${ }^{+}$dendritic cell (DC) accumulation and cystic remodeling of the lung. PLCH typically manifests as a single-system disorder with pulmonary impairment ranging from asymptomatic disease to life-threatening respiratory failure (1) and is pathophysiologically distinct from the LCH of children that often results in visceral infiltration, bone lesions, and multisystem involvement (2). PLCH in adults occurs almost exclusively in active smokers, with an estimated prevalence of 0.27 and 0.07 per 100,000 population in males and females, respectively, and represents up to $5 \%$ of the interstitial lung diseases seen in referral centers $(3,4)$. Little is known about the pathogenesis of PLCH, which was long felt to be a reactive scarring process driven by tobacco use. The discovery over 2 decades ago of recurrent PLCH in the allografts of patients with PLCH who had undergone lung transplantation, however, suggested a metastatic mechanism (5). The recent identification of several activating mutations in the mitogen-activated protein kinase (MAPK) pathway in more than $70 \%$ of patients with PLCH $(6,7)$ is consistent with a metastatic model and suggests that PLCH may be most appropriately classified as an inflammatory neoplastic disorder $(8,9)$. The most common mutation identified to date has been a V600E 
substitution in BRAF kinase (BRAF-V600E), a node in a signaling pathway that plays an essential role in mediating cellular differentiation, proliferation, senescence, and survival in response to extracellular cues (10). Several neoplasms have been reported to harbor BRAF-V600E mutations, including melanoma, lung cancer, and thyroid cancer $(11,12)$. A recent study suggested that constitutive expression of the BRAF-V600E mutation in CD11 $\mathrm{c}^{+} \mathrm{DCs}$ in mice drives a systemic LCH phenotype that is similar to the pediatric form of the disease and that differential expression in the more and less differentiated DC lineages may play a role in the pathogenesis of low- versus high-risk forms of clinical LCH, respectively (10). Recent studies in patients with LCH provide convincing evidence that the mutated cells are most consistent with immature myeloid precursors, that mutations may occur at any point in the myeloid lineage (including monocytes and macrophages) and result in similar effector functions, and that the disease is most appropriately categorized as a myelomonocytic neoplasm.

As efficient antigen-presenting cells, DCs play a pivotal role in the onset and regulation of innate and adaptive immune responses and the recruitment of a variety of leukocytes to the site of inflammation or injury (13). The dysregulation of DC function likely contributes to pathological immune responses and disease pathogenesis in many pulmonary disorders (14). Focal langerin ${ }^{+} \mathrm{DC}$ accumulation around bronchioles and in the pulmonary parenchyma is a key diagnostic feature of PLCH (1). The distribution of these lesions in the upper lobes of the lung (the region most prominently affected by cigarette smoke-induced emphysema) and the recent discovery of DC lineage-specific mutations in PLCH strongly suggest a combinatorial role for smoking-related factors and DCs in the pathogenesis of PLCH. DC migration is tightly regulated by multiple chemokines. Immature DCs express CCR6 and migrate toward the CCL20 ligand, expressed by airway epithelial cells (15). As DCs mature, CCR6 expression diminishes and CCR7 expression predominates (16). Mature DCs then exit the lung and migrate to the draining lymph nodes along a CCR7 ligand-driven chemokine gradient of CCL19 and CCL21 (17). It has been demonstrated that cigarette smoke (CS) affects DC numbers and maturity by increasing the expression of several proinflammatory mediators in the lung, both in humans and in mouse models of chronic obstructive pulmonary disease (1820). However, the effects of CS on DC function and pulmonary accumulation in PLCH are unknown (21).

The objective of this study was to further our understanding of PLCH pathogenesis, including the mechanisms underlying CS-driven recruitment of mutant myeloid cells to the lung and destructive tissue remodeling, using a potentially novel mouse model of inducible, CD11c-specific BRAF-V600E mutations and chronic CS exposure. The model replicates many features of $\mathrm{PLCH}$, including peribronchial and perivascular inflammatory lesions, the development of cellular nodules, and the presence of pulmonary airspace destruction and dilation. Multiple DC functions are altered by the BRAF-V600E mutations, including increased resistance to apoptosis, increased CCL20-dependent pulmonary recruitment and retention, and high levels of expression of the CCL7 chemokine. Further, we found evidence of augmented CCL7 release from the peripheral blood CD11 ${ }^{+}$leukocytes of patients with PLCH. Together, these data indicate that the CD11c-BRAF-V600E animal model for PLCH is an authentic mimic of the human disease suitable for mechanistic studies and as a useful platform for the development of biomarkers and testing potential therapies.

\section{Results}

BRAF-V600E expression in DCs results in PLCH-like pathologies in mice after exposure to CS. We generated a $\mathrm{PLCH}$ murine model by crossing mice with a tamoxifen-inducible (TAM-inducible) CD11c promoter driving Cre recombinase (22) with transgenic mice harboring a floxed genomic cassette containing the BRAFV600E mutation. Placing the $\mathrm{BRAF}^{\mathrm{VE}}$ mice on TAM-containing chow induced the recombination of the endogenous BRAF locus with the transgenic BRAF-V600E exon in CD11c-expressing cells (mice with the recombined BRAF-V600E locus referred to as $\mathrm{BRAF}^{\mathrm{VE}}$ hereafter). The details of the generation of the mouse model are depicted in Supplemental Figure 1; supplemental material available online with this article; https://doi.org/10.1172/jci.insight.132048DS1. Following 2 weeks of TAM chow, mice were exposed to either filtered air (FA) or CS, and tissues were harvested and analyzed at intervals up to 4 months of exposure (Supplemental Figure 1). The expression of BRAF-V600E mRNA following TAM administration in DCs was confirmed by quantitative real-time PCR (Supplemental Figure 1).

$\mathrm{BRAF}^{\mathrm{VE}}$ mice were viable and developed normally without obvious phenotypic alterations compared to WT littermates, with the exception that approximately $20 \%$ of the mice developed splenomegaly/hepatomegaly or small foci of airway epithelial cell hyperplasia between 4 and 6 months of age. BRAF ${ }^{\mathrm{VE}}$ mice 
developed mild peribronchial and perivascular inflammatory infiltrates in the lung that were more prominent after CS exposure (Figure 1A). CS-exposed $\mathrm{BRAF}^{\mathrm{VE}}$ mice also developed parenchymal pulmonary nodules and exhibited heterogenous alveolar destruction and airspace dilation with formation of cyst-like structures (Figure 1A). Control mice exposed to FA or CS did not develop any of these pathologies. Three of 8 FA-exposed BRAFVE mice did develop some nodules, but they were fewer in number and smaller in volume, individually and in aggregate, compared with those in the CS-exposed BRAFVE mice (Figure 1B). Similarly, only CS-exposed $\mathrm{BRAF}^{\mathrm{VE}}$ mice developed the cyst-like structures in the lung (Figure 1C). These pathologies are CS dependent because the $\mathrm{BRAF}^{\mathrm{VE}}$ mice have normal alveolar architecture at birth and before CS exposure. Immunohistochemical (IHC) studies revealed that the nodules consisted of centrally located myelomonocytic cells as defined by CD68 expression and peripherally distributed lymphocytes, including T cells and B cells (Figure 1D and ref. 6). These findings recapitulate the hallmark characteristics of airspace enlargement and inflammatory nodules that define human PLCH. More importantly, our data demonstrate that CS exacerbates the PLCH phenotype in mice, supporting the concept that CS is not only a demographic feature of PLCH but also a cofactor that is required for full expression of disease pathogenesis in the context of myelomonocytic BRAF-V600E mutations.

$B R A F^{V E}$ mice exhibit pulmonary inflammation and disrupted DC homeostasis in the lung following CS exposure. To examine the populations and phenotypes of cells recruited to the lungs of BRAFVE mice exposed to CS, we examined single-cell suspensions obtained by whole-lung enzymatic digestion using flow cytometric analysis. There was an increase in DCs and T cells but not macrophages (Figure 2A), suggesting that the $\mathrm{CD} 8^{+}$histiocytes that accumulate in the nodules may have a DC origin (23). Mouse CD103+ DCs express langerin similar to that of the human CD1a+ DCs found in PLCH (24). Examination of the major populations of conventional DCs in $\mathrm{BRAF}^{\mathrm{VE}}$ mouse lungs revealed that CS exposure specifically increased the accumulation of $\mathrm{CD}_{103^{+}}$and $\mathrm{CD} 11 \mathrm{~b}^{+} \mathrm{DCs}$ (Figure 2B). Moreover, BRAF ${ }^{\mathrm{VE}}$ mice exhibited an increase in $\mathrm{CD}^{+} 6^{+}$mature DCs in the lungs that was further increased by CS exposure (Figure $2 \mathrm{C}$ ) analogous to DC accumulation in patients with PLCH (25). Next, we assessed the functional effects of BRAF-V600E mutation and CS exposure. Pulmonary DCs isolated from BRAFVE mice secreted high levels of proinflammatory cytokines IL-6 and IL-12 upon stimulation with agonists for TLR-2 [poly(I:C)] and TLR-4 (LPS) compared with WT mice. This increased responsiveness was potentiated in DCs from BRAFVE mice exposed to CS compared with FA (Figure 2, D and E). Taken together, these data support a model in which aberrant DC function in PLCH is a consequence of both dysfunctional signaling due to the BRAF-V600E mutation and CS exposure.

BRAF-V600E DCs demonstrate increased activation, cell viability, and expression of the antiapoptotic factor $B$ cell lymphoma leukemia-x molecule. We sought to determine whether DC accumulation in the lungs of $\mathrm{BRAF}^{\mathrm{VE}}$ mice is due to increased activation and proliferation of DCs, inhibition of apoptosis, or both. Bone marrow-derived dendritic cells (BMDCs) from BRAFV600 $\mathrm{E}^{\mathrm{fl} / \mathrm{fl}}$ transgenic mice were transfected ex vivo with adenovirus expressing Cre recombinase to generate mutant DCs (referred to as BRAFVE BMDCs) (Supplemental Figure 2, A-D). BRAF ${ }^{\mathrm{VE}}$ BMDCs exhibited enhanced ERK phosphorylation consistent with constitutive activation of the MAPK pathway that was completely inhibited by the BRAF-V600E inhibitor PLX4720 (Figure 3A). Although constitutive activation of the MAPK pathway by BRAF-V600E is typically associated with increased cell proliferation and attenuation of apoptosis (26), DCs are more terminally differentiated cells that may not respond in the same manner as cells from other lineages. To determine whether the BRAF-V600E mutation affects DC proliferation, we counted cultured BMDCs over time following transfection and observed no differences in proliferation between WT and BRAF ${ }^{\mathrm{VE}}$ BMDCs (Figure 3B). MAPK signaling is also known to regulate apoptosis under different physiological and neoplastic conditions $(27,28)$. Therefore, we next examined the effects of the BRAF-V600E mutation on DC viability. Cre-transfected WT and BRAFVE BMDCs were collected at day 7 of differentiation and cultured in the absence of the DC survival-dependent growth factor GM-CSF for 24 hours. Flow cytometric analysis illustrated that the nonapoptotic population (propidium iodidenegative $\left[\mathrm{PI}^{-}\right]$, annexin $\mathrm{V}^{-}$) of $\mathrm{BRAF}^{\mathrm{VE}}$ BMDCs was increased compared with WT BMDCs (Figure $3 C)$. To further investigate the mechanism of enhanced $B R A F^{V E} D C$ viability, we measured BMDC expression of antiapoptotic protein B cell lymphoma leukemia-x molecule (Bcl-xL). Consistent with the observed reduction in apoptosis, the expression of Bcl-xL in BRAFVE BMDCs was increased compared with WT (Figure 3D). Examination of DCs from BRAF ${ }^{\mathrm{VE}}$ mice demonstrated a consistent increase in the survival capacity of DCs compared with WT (Figure 3E). 
A

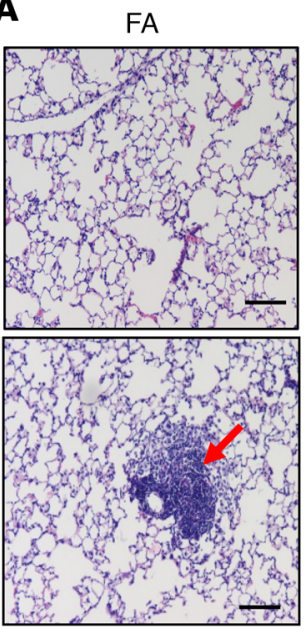

CS

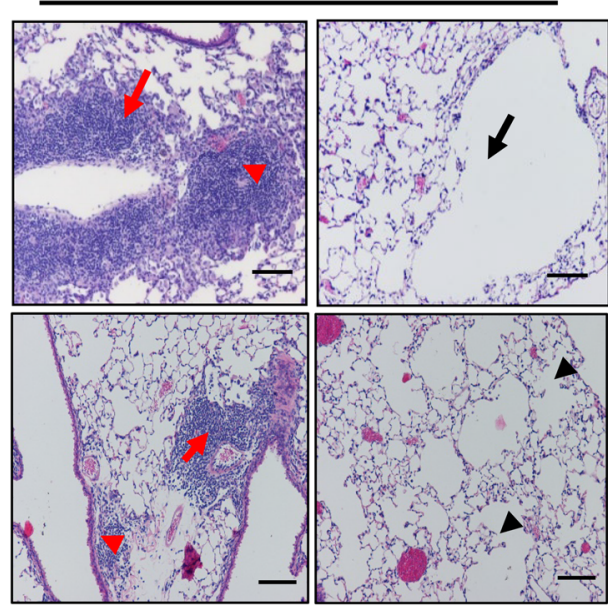

B

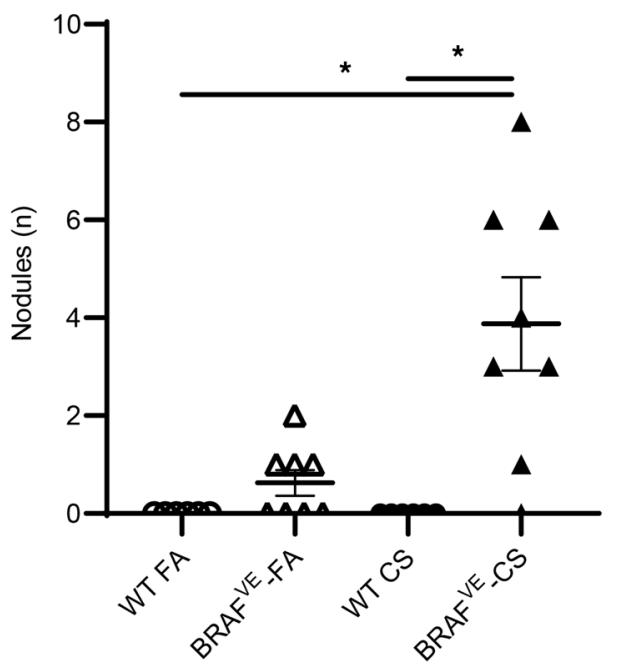

C

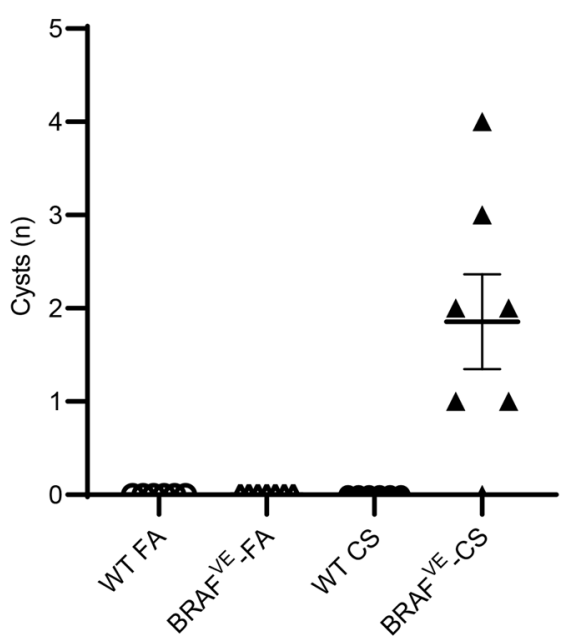

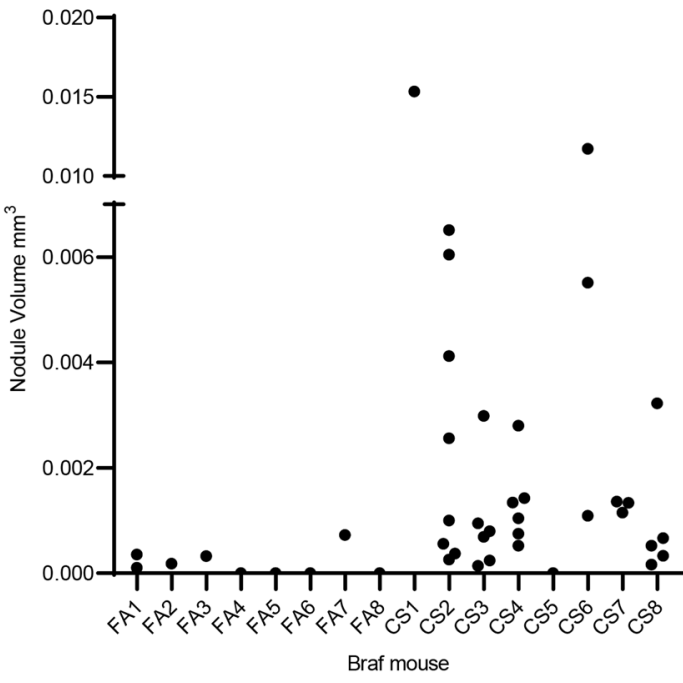

D
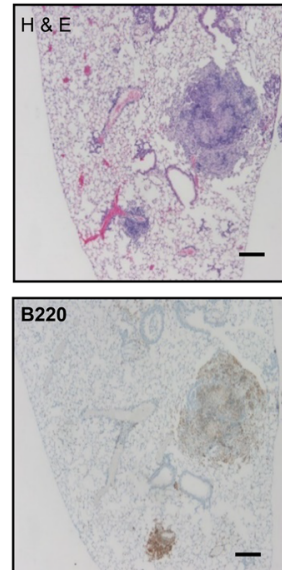

Figure 1. Exposure of mice with BRAF-V600E expression in CD11c ${ }^{+} \mathrm{DCs}$ to CVS results in PLCH-like lesions. (A) Representative H\&E-stained sections showing lung pathology in BRAFVE mice exposed to FA or CS for 4 months. Red arrowheads indicate parenchymal nodule formation and red arrows indicate perivascular and peribronchiolar cell infiltration. Black arrows indicate cyst-like lesions and black arrowheads indicate areas of heterogeneous alveolar tissue destruction. Scale bar: $100 \mu \mathrm{m}$. Representative image of $n=8$ mice per group. (B) Number and volume of pulmonary nodules. Nodules were identified as large inflammatory cell infiltrates not surrounding an airway or vessel. The volume of each individual nodule was estimated as described in Methods. $n=8$ mice per group. (C) Enumeration of pulmonary cystic structures more than $200 \mu \mathrm{m}$ in diameter. Representative lesions from $n=$ 8 mice per group. (D) Representative IHC staining of nodular inflammatory lesions in lungs of BRAF $^{\mathrm{VE}}$ mice exposed to CS for 4 months. Scale bar: $250 \mu \mathrm{m}$. Representative lesions from $n=$ 8 mice per group. For experiments shown, 1-way ANOVA with Tukey's multiple-comparisons analysis was performed. ${ }^{*} P<0.05$. 
A
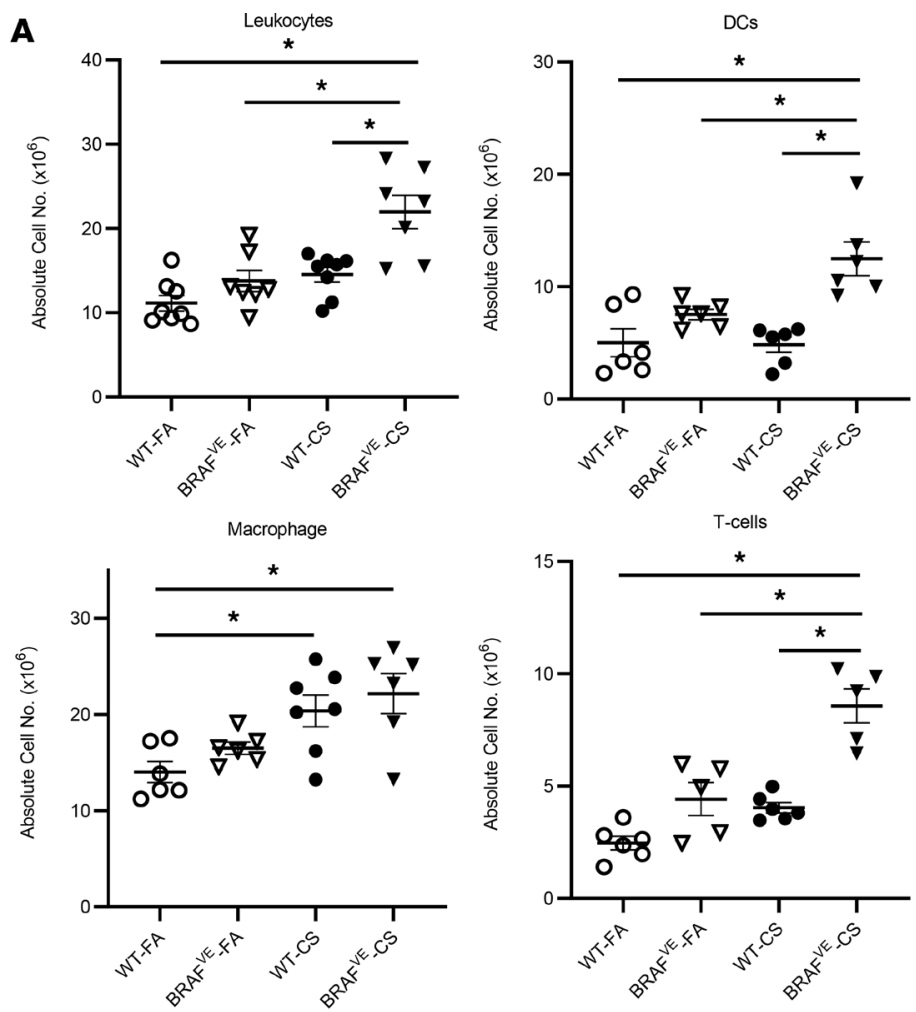

B
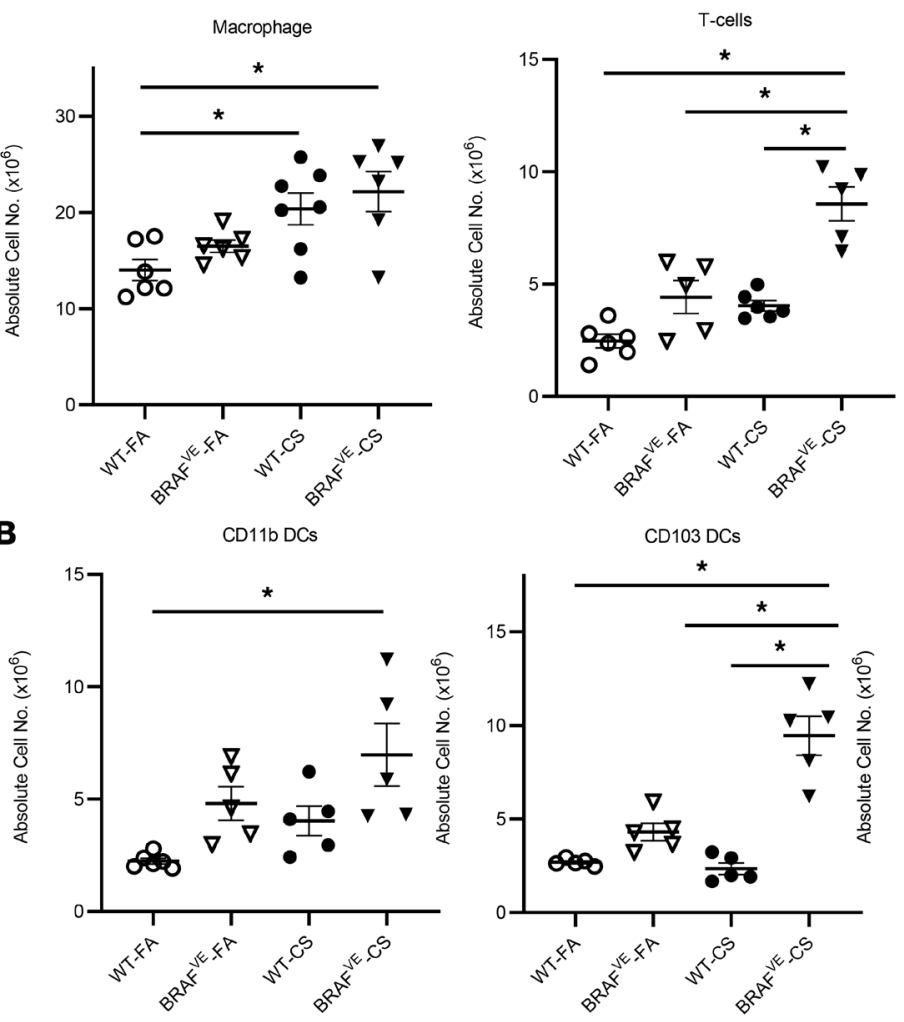

D

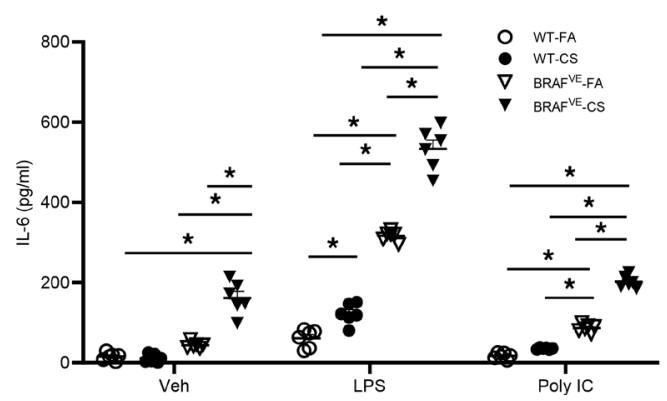

E

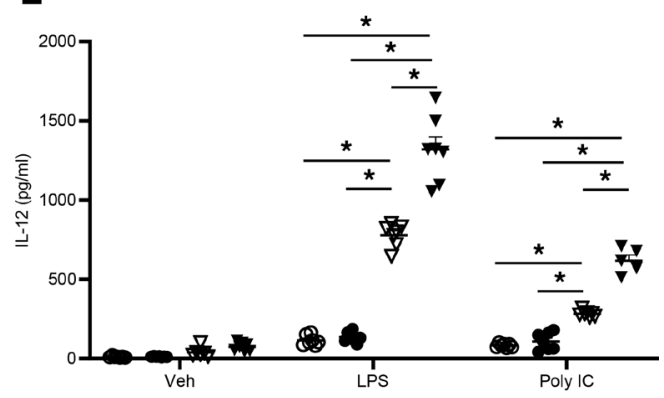

Figure 2. Increased inflammatory cells and disrupted DC homeostasis in the lungs of BRAF ${ }^{\mathrm{VE}}$ mice. (A) Absolute cell numbers of leukocytes, DCs, macrophages, and T cells in the dissociated lungs of WT ( $n$ $=5-8$ mice per group) and $\operatorname{BRAF}^{\mathrm{VE}}(n=5-7$ mice per group) mice were determined by flow cytometry. Leukocytes were identified as $\mathrm{CD} 45^{+}$; DCs were identified as CD11C ${ }^{+}$, MHC $\mathrm{II}^{+}$, and autofluorescence ${ }^{\mathrm{mid} /}$ low cells; macrophages were identified as $\mathrm{CD}_{11 \mathrm{c}^{+}}$and autofluorescence ${ }^{\text {hi }}$ cells: and $T$ cells were identified as CD11c ${ }^{-}$and $\mathrm{CD}^{+}{ }^{+}$cells ( $n=$ 5 mice per group). (B) The absolute numbers of CD11 ${ }^{+}$ $\mathrm{DCs}$ and $\mathrm{CD}_{103^{+}} \mathrm{DCs}$ were determined by flow cytometry. Both subsets were gated from the DC population. (C) The number of DCs expressing maturation marker CD86 was determined by flow cytometry ( $n$ = 5 mice per group). ( $\mathbf{D}$ and E) DCs were isolated from lungs and treated with or without $20 \mathrm{ng} / \mathrm{mL}$ IFN- $\gamma$ for 2 hours before being treated with $1 \mu \mathrm{g} / \mathrm{mL}$ poly(I:C) or 1 $\mu \mathrm{g} / \mathrm{mL}$ LPS for 16 hours. The supernatant was collected and IL-6 and IL-12 p40 were measured by ELISA ( $n=$ 5-7 mice per group). For experiments shown, ANOVA (1 way in $\mathbf{A}$ and $\mathbf{B}, 2$ way in $\mathbf{D}$ and $\mathbf{E}$ ) with Tukey's multiple-comparisons analysis was performed. ${ }^{*} P<0.05$. Data represent mean \pm SEM. 


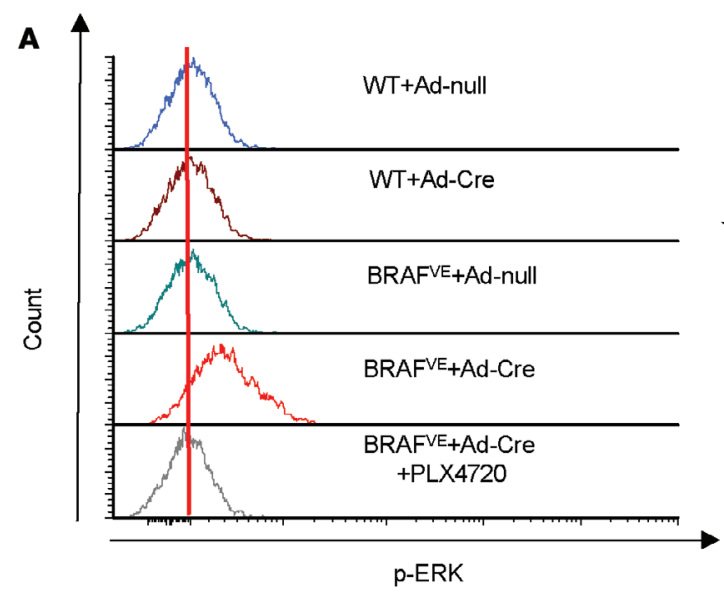

C

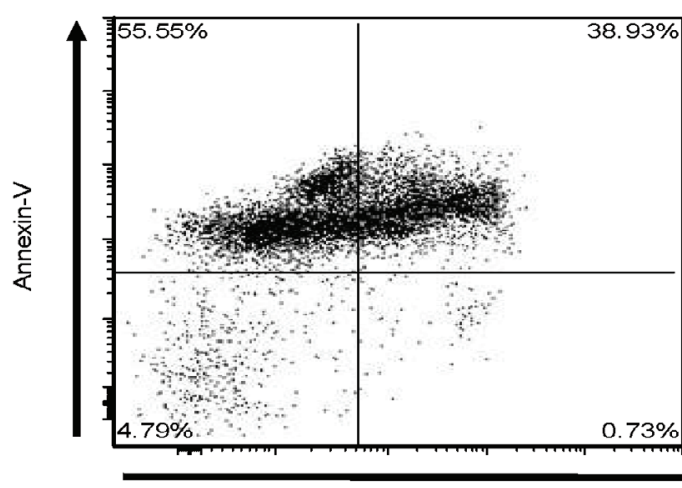

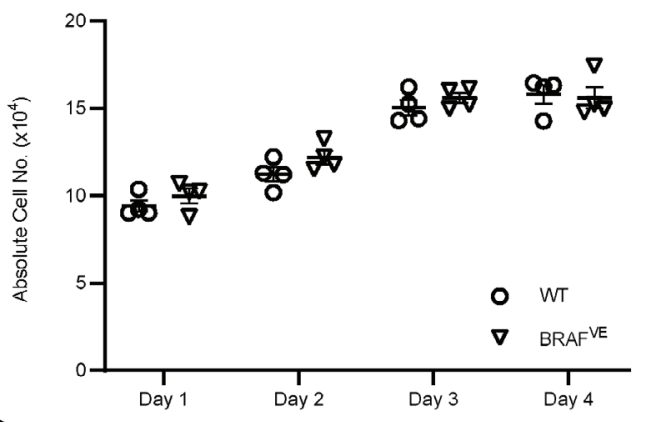

BRAFVE

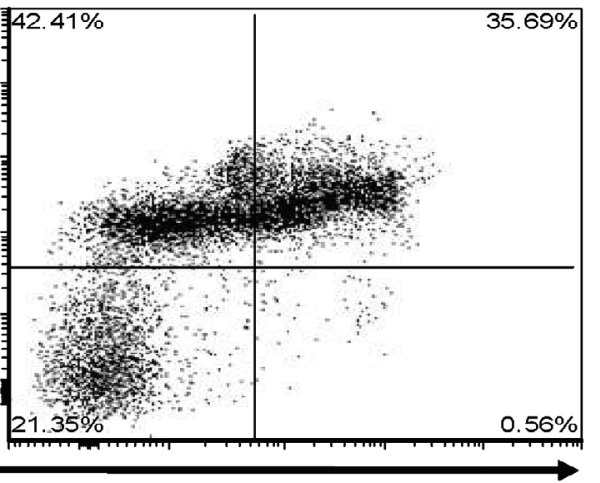

PI

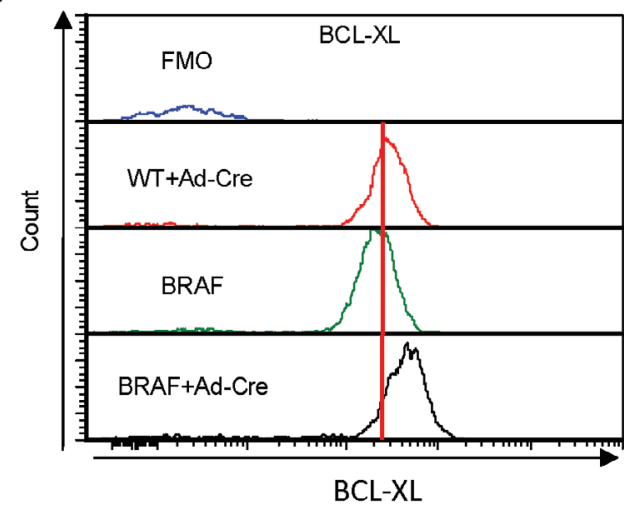

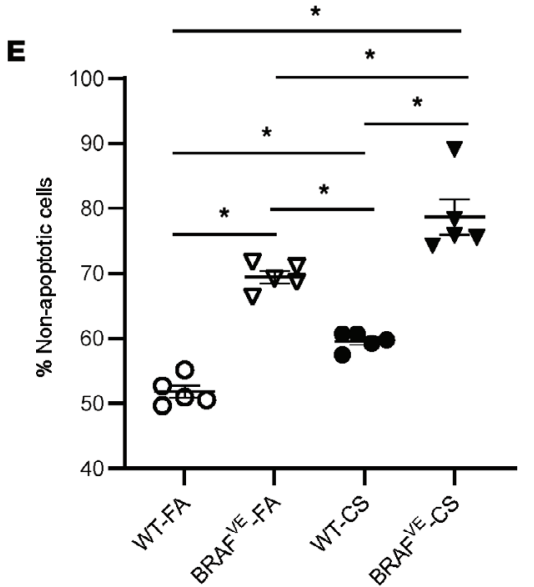

Figure 3. BRAF-V600E mutation is associated with increased cell viability and expression of the antiapoptotic protein B cell lymphoma leukemia-x molecule. (A) Phospho-ERK ( $p$-ERK) expression in BMDCs from WT and BRAFV-600E mice following the administration of Cre Recombinase Adenovirus mCherry (Ad-Cre) to induce BRAFV-600E expression was determined by flow cytometry. In indicated groups, $1 \mu$ M PLX4720 (BRAF inhibitor) was added to the BMDC culture at day 6 . Data shown are representative of 4 independent experiments. (B) Absolute number of BMDCs ( $n=4$ WT/day; $n=4 \mathrm{BRAF}^{\mathrm{VE}} /$ day) determined by flow cytometry at the indicated times. (C) BRAFV-600E expression increases BMDC viability in vitro. GM-CSF was removed from the culture medium at day 7, and apoptosis was quantified by annexin $V$ and PI staining after 16 hours using flow cytometry. Live cells are annexin $\mathrm{V}^{-}$and $\mathrm{PI}^{-}$. Data shown are representative of 4 independent experiments. (D) BRAFV-600E expression increases antiapoptotic protein Bcl$\mathrm{xL}$ expression in vitro. The expression of the antiapoptotic marker $\mathrm{Bcl}-\mathrm{xL}$ in BMDCs was measured by flow cytometry. Data shown are representative of 4 independent experiments. (E) BRAFV-600E expression increases CD11c cell viability in vivo. CD11c cells were isolated from WT and BRAFVE mice, and the viability was assessed by flow cytometry as described above. Data are shown as the mean $\pm \mathrm{SEM} ; n=4$ per each group. For the experiments shown, B and $\mathbf{E}$ used 1-way ANOVA with Tukey's multiple-comparisons test. Data shown are mean \pm SEM. ${ }^{*} P<0.05$. 
BRAF-V600E mutation is associated with increased CCL20 production and CCR6 responsiveness in DCs. Along with increased cell viability, a potential alternative or complementary mechanism for pulmonary DC accumulation is increased DC recruitment from the circulation. The lung-resident DC pool is replenished by circulating DCs and pre-DCs at a constant rate under steady-state conditions. DC recruitment can be rapidly increased during inflammation by directed migration toward the chemokine CCL20 (17). We examined CCL20 expression in lung biopsies of healthy controls and patients with PLCH. Lung tissues showed diffuse CCL20 staining in the PLCH lesions compared with healthy controls as well as intense staining of histiocyte-like cells (Figure 4A). We also measured CCL20 levels in bronchoalveolar lavage (BAL) fluid collected from WT and BRAFVE mice exposed to FA and CS. BAL CCL20 levels were increased in $\mathrm{BRAF}^{\mathrm{VE}}$ mice compared with $\mathrm{WT}$ and were further increased in $\mathrm{BRAF}^{\mathrm{VE}}$ mice that were exposed to CS (Figure 4B). Because the CCL20 expression pattern in PLCH lung tissue indicated that CCL20 may be derived from histiocytes within lung lesions, we assessed the capacity of BRAF ${ }^{\mathrm{VE}}$ BMDCs to produce CCL20. No CCL20 production was observed in unstimulated WT BMDCs whereas $\mathrm{BRAF}^{\mathrm{VE}}$ BMDCs secreted significant amounts of CCL20 (Figure 4C). Further, these studies showed that LPS activation of BRAFVE BMDCs potentiated CCL20 production.

We next examined whether the BRAF-V600E mutation affects BMDC responsiveness to CCL20. The sole receptor for CCL20, CCR6, is a G-coupled transmembrane spanning protein that inhibits intracellular cAMP following receptor activation (29). We evaluated CCR6-dependent CCL20 responsiveness by measuring cellular cAMP levels upon stimulation with CCL20. Our data showed that CCL20 reduced forskolin-stimulated cAMP levels more potently in $\mathrm{BRAF}^{\mathrm{VE}} \mathrm{BMDC}$ s compared with WT BMDCs, indicative of increased CCR6 responsiveness (Figure 4D). In contrast, evaluation of CCR7 responsiveness, as defined by inhibition of cAMP levels following CCL19 and CCL21 stimulation, was not different between WT and BRAFVE BMDC (Supplemental Figure 3). Consistent with this finding, we also observed enhanced functional responses to CCL20 as measured by increased migration of BRAFVE BMDCs compared with WT BMDCs toward CCL20, but not CCR7, CCL19, or CCL21, using a transwell migration assay (Figure 4E and Supplemental Figure 3). We postulated that the altered lung microenvironment is a key factor in the recruitment, accumulation, and activation of BRAF ${ }^{\mathrm{VE}}$ DCs in PLCH. To examine the effects of CS exposure on DC accumulation in the lung, we assessed the migratory capacity of $\mathrm{BRAF}^{\mathrm{VE}} \mathrm{BMDCs}$ by adoptive transfer. Donor WT or $\mathrm{BRAF}^{\mathrm{VE}} \mathrm{BMDCs}$ derived from CD45.2 donor mice were injected into the tail vein of CD45.1 recipient mice that were preexposed to FA or CS. Lungs and mediastinal lymph nodes (mLNs) were collected from recipient mice 2 days after injection, and the retention of donor BMDCs in these tissues was analyzed by flow cytometry. These studies showed that BRAFVE BMDC accumulation in the lung was greater than WT BMDCs and was further increased in the CS-exposed recipients (Figure 4F). There was no difference in the abundance of $\mathrm{WT}$ and BRAF ${ }^{\mathrm{VE}}$ BMDCs in mLNs. The findings that both the BRAF-V600E mutation and CS exposure are required for PLCH-like lesion development is further supported by greater pulmonary accumulation of adoptively transferred BRAF ${ }^{\mathrm{VE}}$ DCs than WT DCs and by the greater accumulation of adoptively transferred $\mathrm{BRAF}^{\mathrm{VE}}$ DCs in CS- than FA-conditioned recipients. Together, these studies establish that the CS-dependent accumulation of DCs in the lung of $\mathrm{BRAF}^{\mathrm{VE}}$ mice is due to the combination of enhanced cell viability and recruitment of BRAF-mutant cells and not due to enhanced proliferation.

CCL7 chemokine expression is increased in BRAFVE mice and is associated with the BRAF-V600E mutation in DCs. In addition to DCs, inflammatory lesions in $\mathrm{PLCH}$ and $\mathrm{BRAF}^{\mathrm{VE}}$ mice consist of many leukocytes, including macrophages, T cells, B cells, and eosinophils (6). We postulated that mutant DCs orchestrate the recruitment of other cells to the lesion in a BRAF-V600E-dependent manner. Using multiplex ELISA, we probed the expression of several DC-related chemokines and cytokines in serum (Supplemental Figure 4). We found that CCL7, a pluripotent chemokine that can bind to receptors expressed on T cells, monocytes, eosinophils, and NK cells (30), was present at very low levels in WT mice exposed to FA or CS but was increased in $\mathrm{BRAF}^{\mathrm{VE}}$ mice independent of CS exposure, suggesting a direct link between the BRAF mutation and CCL7 production (Figure 5A). Because the CCL7 expression pattern appeared to be BRAF-V600E dependent, we examined the possibility that the BRAF ${ }^{\mathrm{VE}}$ DCs were the source of CCL7 in our model. First, we isolated pulmonary CD11 $\mathrm{c}^{+}$cells and measured CCL7 secretion with and without LPS activation. We found that $\mathrm{BRAF}^{\mathrm{VE}}$ cells produced significantly greater levels of CCL7 compared with WT and that CCL7 secretion from DCs isolated from BRAF ${ }^{\mathrm{VE}}$ mice increased following activation (Figure 5B). The BRAF-V600E-specific inhibitor, PLX4720, decreased CCL7 expression in these cells, further implicating BRAF in the induction of CCL7 expression (Figure 5B). The association between BRAF-V600E and CCL7 expression was supported 

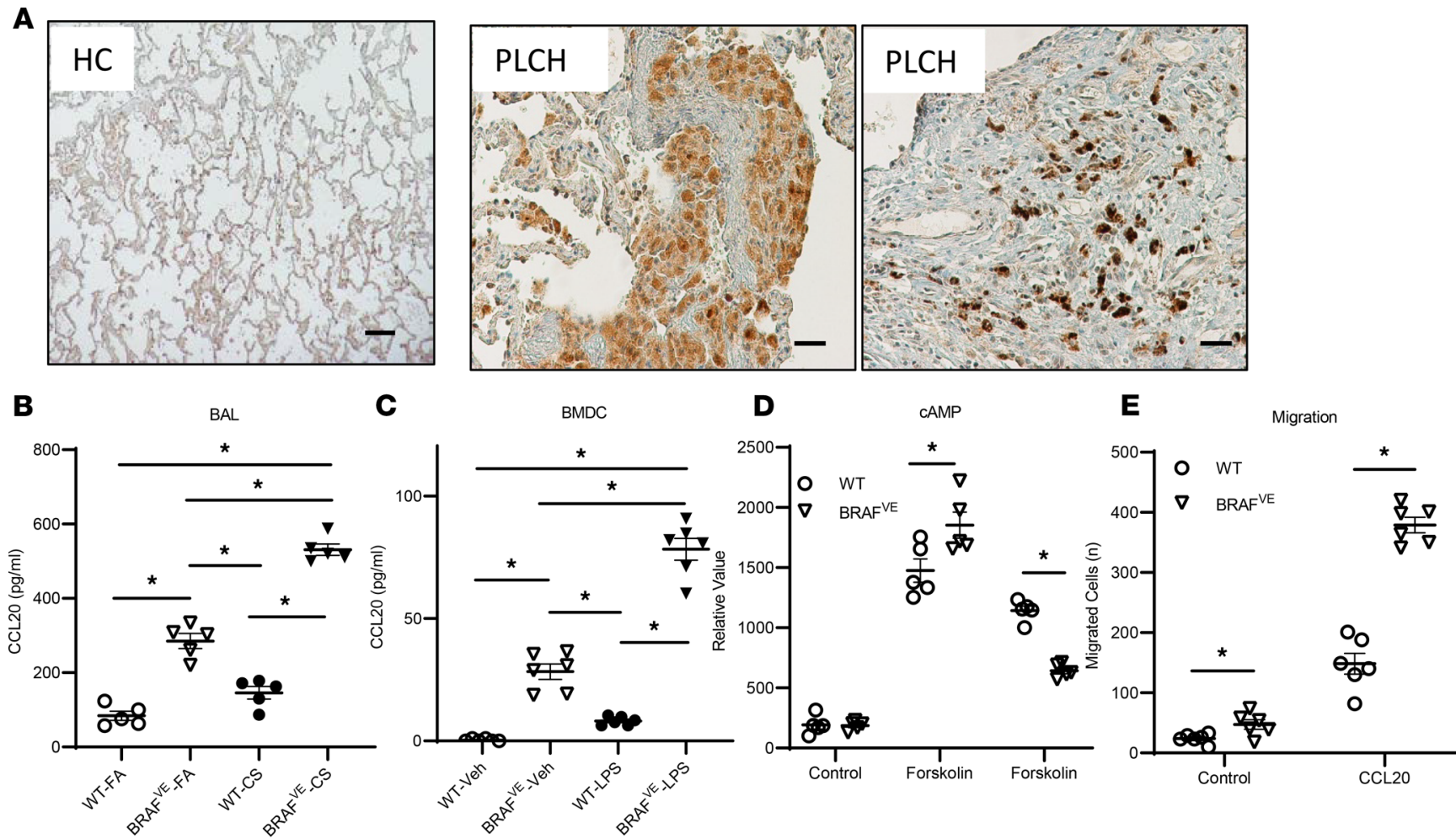

$\mathbf{F}$

FA
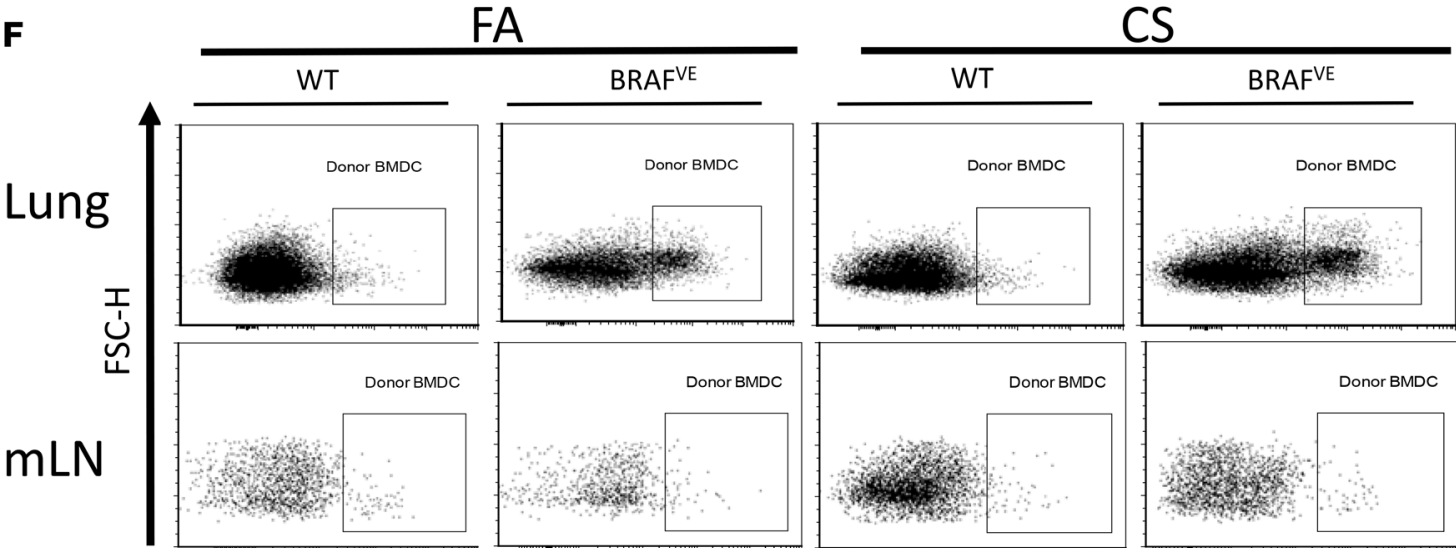

\section{CD45.2}
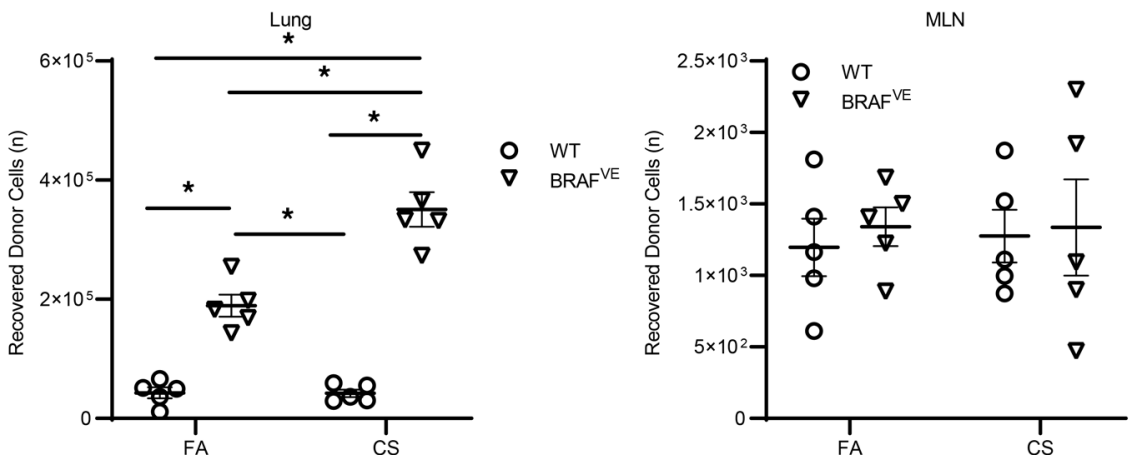
Figure 4. BRAF-V600E mutation increases the recruitment of DCs to the lung in a CCL20-dependent manner. (A) Representative IHC staining for CCL20 in lung sections from healthy controls (HC) and patients with PLCH ( $n=3 /$ group). Scale bar: $100 \mu \mathrm{m}$. (B) CCL20 concentration in the BAL of mice ( $n=5$ /group) exposed to FA or CS for 4 months was measured by ELISA. Data shown are mean \pm SEM of 5 independent experiments. (C) CCL20 secretion by WT or BRAF ${ }^{\mathrm{VE}}$ donor BMDCs ( $n=6$ /group) treated with/without LPS plus IFN- $\gamma$ was measured by ELISA. Data are representative of 5 independent experiments. (D) Intracellular cAMP levels in WT or BRAFVE BMDCs ( $n=5 /$ group) were measured after treatment with forskolin followed by CCL20 stimulation $(300 \mathrm{ng} / \mathrm{mL})$. Data are representative of 3 independent experiments. (E) The number of WT or BRAFVE BMDCs that migrated from the upper to lower chamber of a Transwell plate toward CCL20 $(300 \mathrm{ng} / \mathrm{mL})$ was determined by flow cytometry after 3 hours $(n=6 /$ group). Data are representative of 3 independent experiments. (F) Two million WT or BRAF ${ }^{\mathrm{VE}}$ CD45.2 donor BMDCs were intravenously injected into recipient CD45.1 mice that had been previously exposed to FA/CS for 6 months. The lungs were harvested 2 days later, and the numbers of donor cells in the lung and $\mathrm{mLNs}$ of the recipient mice were determined by gating on CD45.2 expression. Data shown are mean \pm SEM of 3 independent experiments. (B-F) ${ }^{*} P<0.05$, ANOVA ( 1 way in $\mathbf{B}$ and $\mathbf{C}, 2$ way in $\mathbf{D}-\mathbf{F}$ ) with Tukey's multiple-comparisons test. Data shown are mean \pm SEM.

in BRAF ${ }^{\mathrm{VE}} \mathrm{BMDCs}$ by immunofluorescence staining for CCL7 (Figure 5C). The link between BRAF-V600E and CCL7 expression was further demonstrated in the BRAF ${ }^{\mathrm{VE}}$ BMDCs, which showed that mutant DC expression of CCL7 was detectable at baseline, upregulated by DC stimulation, and almost completely inhibited following BRAF-V600E inhibition with PLX4720 (Figure 5D). In contrast, although CCL2 levels (another pluripotent cytokine that shares several receptors with CCL7) were measurable at baseline and increased by DC activation (Figure 5E), there was no difference between WT and mutant DCs, and BRAF inhibitors did not attenuate CCL2 expression. Identical patterns of CCL7 and CCL2 expression were also evident at the transcriptional level (Figure 5F). The BRAF/CCL7 axis was further explored using the A-375 melanoma cell line harboring the BRAF-V600E mutation. Interestingly, the melanoma cell line was incapable of secreting CCL7, suggesting that the cell lineage is also a critical determinant of function in the context of the BRAFV600E mutation (Supplemental Figure 5).

Given the stark increase and specificity for serum CCL7 in the mouse model of PLCH, we measured serum levels of CCL7 in patients with PLCH with unknown MAPK/BRAF mutations. These results demonstrate that CCL7 is similarly elevated in patients with PLCH compared to healthy never smokers and current smokers (Figure 6A). We further examined CCL7 expression levels in PLCH patient samples using flow cytometry. We obtained PBMCs from 3 patients with PLCH with confirmed BRAF-V600E mutations. Activation of PBMCs from 2 of the $3 \mathrm{~V} 600 \mathrm{E}$ patients, but not healthy controls, revealed unique populations of $\mathrm{CD} 11 \mathrm{c}^{+}$cells expressing CCL7 (Figure 6B). Together, these results suggest that the recruitment of inflammatory cells other than $\mathrm{BRAF}^{\mathrm{VE}} \mathrm{DC}$ into the stroma of PLCH lesions is a secondary event driven by CCL7 secreted from the mutant DCs.

Withdrawal of CS and TAM ameliorates the PLCH phenotype in the BRAFVE mice. We have shown that both the CS-conditioned local pulmonary environment and the BRAF-V600E mutation are important for the pathogenesis of PLCH-like lesions in this mouse model. Therefore, cessation of CS exposure and blocking aberrant signaling by BRAF mutants provide potential therapeutic strategies. To investigate the efficacy of this combined approach, we discontinued CS exposure and TAM treatment of the BRAFVE mice after 4 months of exposure to both (Figure 7A and Supplemental Figure 6). In addition, CCL7 and CCL20 levels in BAL fluid from BRAFVE mice reverted to baseline after dual cessation (Figure 7B), as did the total number of cells, CD103+ DCs, and T cells (Figure 7C). Taken together, these data indicate that discontinuing exposure to $\mathrm{CS}$ and the withdrawal of TAM were capable of attenuating lung remodeling in BRAFVE mice.

\section{Discussion}

The paucity of cellular and molecular data regarding PLCH pathogenesis is an obstacle to the development of effective clinical therapies. Here, we report the development of a potentially novel animal model of PLCH using mice with an inducible BRAF-V600E mutation in myelomonocytic cells and CS exposure. These mice develop several of the hallmark characteristics of $\mathrm{PLCH}$, including peribronchial inflammatory nodules and cyst-like structures in the lung. Although up to $90 \%$ of patients with PLCH are active smokers, the role of CS in disease pathogenesis has remained a mystery (1). In this study, we show that $\mathrm{CS}$ exposure is central to the development and maintenance of the PLCH lesions. Although BRAF ${ }^{\mathrm{VE}}$ mice exposed to FA develop mild peribronchiolar inflammation, they do not develop the parenchymal nodules or tissue destruction observed in CS-exposed BRAFVE mice. Smoking cessation can lead to improvement in symptoms and radiographic disease resolution in some patients with PLCH (31). Consistent with these clinical observations, the pulmonary phenotype of CS-exposed BRAFVE mice was lessened following cessation of smoke exposure and TAM induction of mutant cells. This observation suggests that CS gener- 

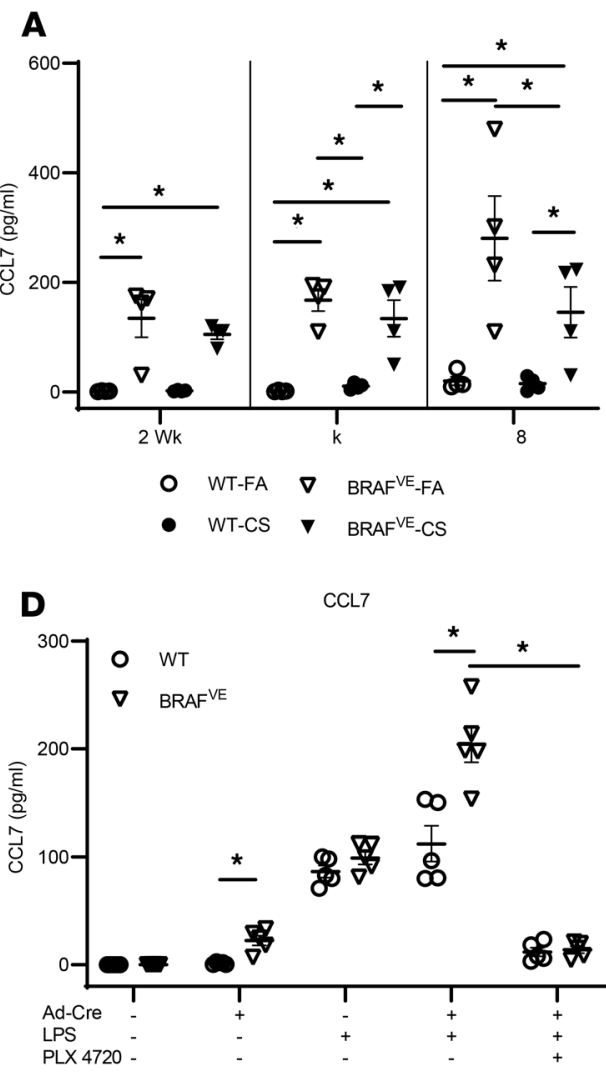

B

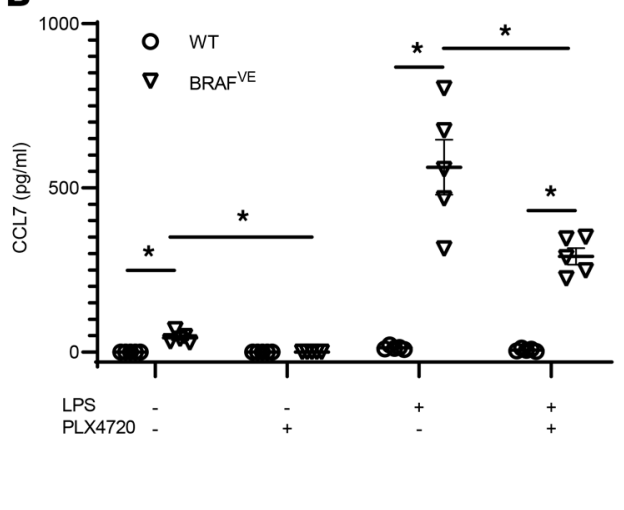

$\mathbf{E}$

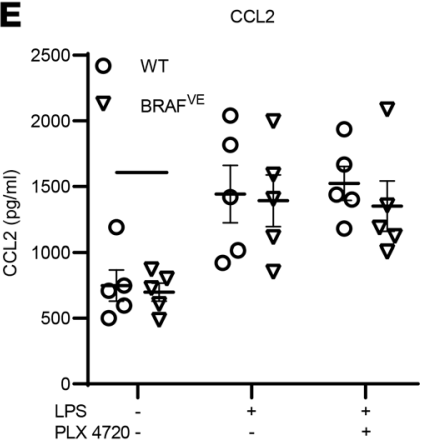

C

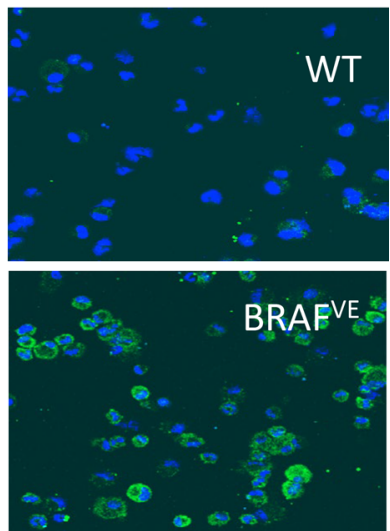

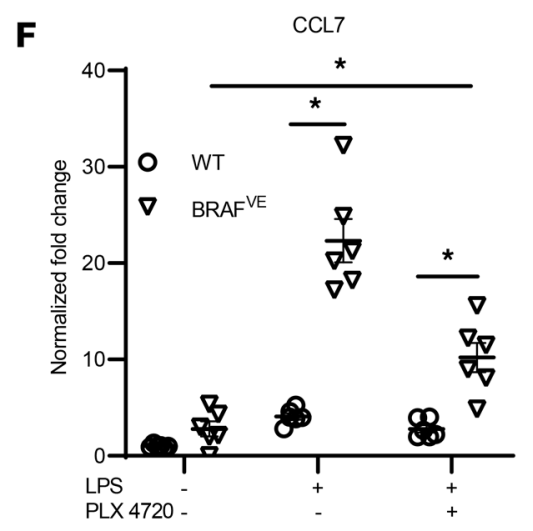

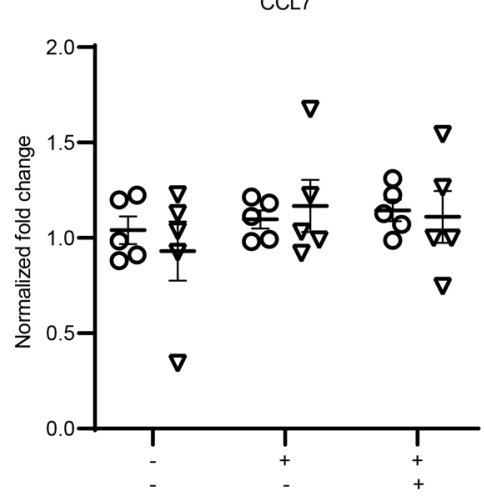

Figure 5. BRAF-V600E mutation induces DCs production of CCL7. (A) Peripheral blood was collected from WT or BRAFVE mice exposed to FA/CS for indicated periods, and the CCL7 level in the serum was measured by ELISA ( $n=4 /$ group). (B) WT or BRAFVE pulmonary DCs were treated with/without $1 \mu \mathrm{g} / \mathrm{mL}$ LPS or $1 \mu \mathrm{M}$ BRAF-specific inhibitor PLX4720 or both, and CCL7 in the supernatant was measured by ELISA ( $n=5 / \mathrm{group}$ ). (C) Representative CCL7 staining of BMDCs ( $n=5 /$ group) from WT or BRAFVE mice treated with $1 \mu \mathrm{g} / \mathrm{mL}$ LPS overnight. Blue, DAPI; green, CCL7. Data are representative of 4 independent experiments. ( $\mathbf{D}$ and $\mathbf{E}$ ) The secretion of (D) CCL7 and (E) CCL2 from WT or BRAFVE BMDCs treated with/without Ad-Cre at 150 multiplicity of infection (MOI), $1 \mu \mathrm{g} / \mathrm{mL}$ LPS and $1 \mu \mathrm{M}$ PLX4720, was determined by ELISA ( $n=5 / \mathrm{group}$ ). Data are representative of 5 independent experiments. (F) CCL7 and CCL2 mRNA expression level in WT or BRAFVE BMDCs treated with/without $1 \mu \mathrm{g} / \mathrm{mL}$ LPS and $1 \mu \mathrm{M}$ PLX4720 was determined by real-time PCR. Data are representative of 3 independent experiments. ${ }^{*} P<0.05,2$-way ANOVA with Tukey's multiple-comparisons test. Data shown are mean \pm SEM.

ates a conditioned microenvironment in the lung that favors the accumulation and/or retention of pathogenic BRAF-V600E DCs. This is further supported by clinical observations that children with extrapulmonary LCH who subsequently develop PLCH during adolescence or adulthood are often smokers $(32,33)$.

We examined several potential mechanisms for the accumulation of mutant histiocytes in the lung, including unchecked proliferation of BRAF-V600E DCs, resistance to apoptosis, and aberrant responsiveness to chemotactic signals that mediate DC migration into and out of the lung. A previous study shows that the DCs in LCH lesions exhibit low proliferative activity $(34,35)$. Consistent with these findings, our data reveal that BRAF-V600E mutation is not associated with increased proliferation 
A

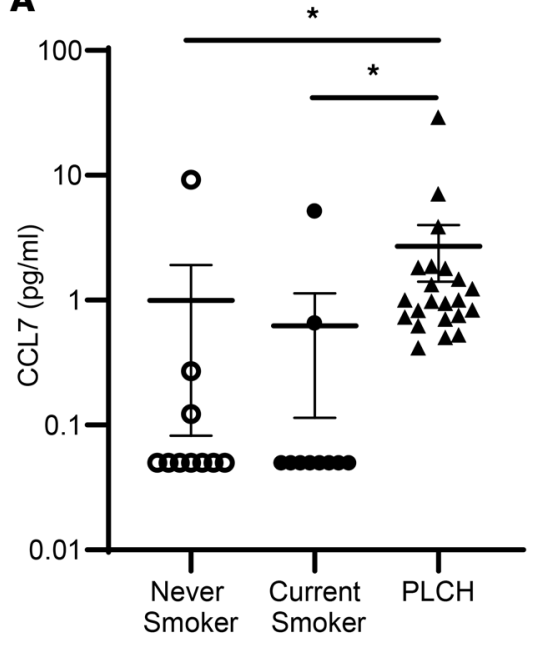

Figure 6. Increased CCL7 in the serum and CD11c ${ }^{+}$PBMCs of PLCH patients. (A) CCL7 levels were measured in the serum of never smokers $(n=10)$, current smokers $(n=10)$, and PLCH patients $(n=22)$ by ELISA. Groups were compared using Kruskal-Wallis 1-way analysis of variance on ranks with Dunn's multiple-comparisons test. ${ }^{*} P<0.05$. (B) PBMCs were isolated from healthy controls and PLCH patients and treated with or without LPS and IFN- $\gamma$ overnight. The CCL7+ cells were identified exclusively in the $\mathrm{CD} 11 \mathrm{c}^{+}$ population of PBMCs by flow cytometry. Representative plots from 3 patients and controls are shown.
B

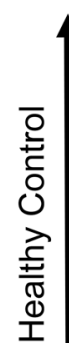

Control

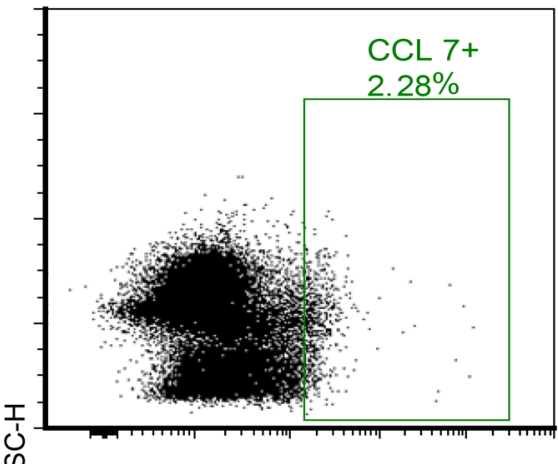

心

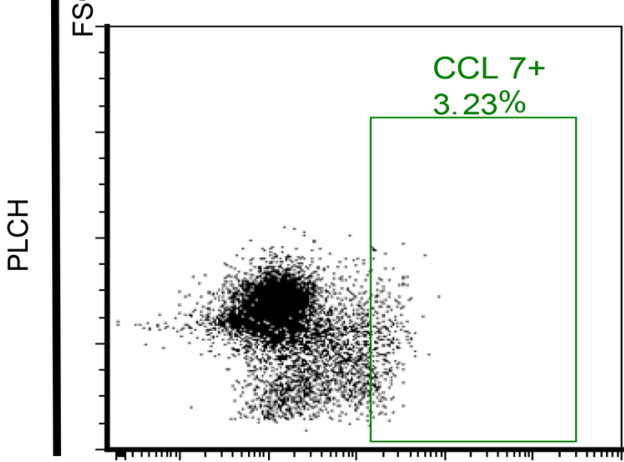

Activated
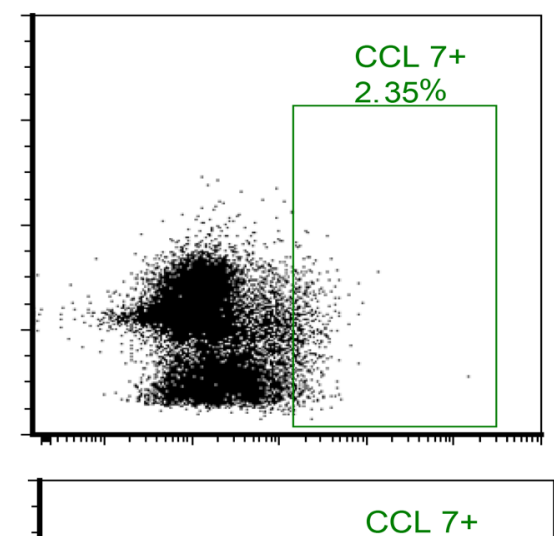

$14.31 \%$

CCL7

in mutant DCs in the PLCH model. Alternative mechanisms of increased cell accumulation include abnormal recruitment, survival, or egress from the lung. It is known that CS exposure promotes survival of DCs by inducing the expression of the antiapoptotic molecule Bcl-xL, which has been shown to be overexpressed in PLCH biopsies (36). Similar to a recent report, we show that the BRAF-V600E mutation is associated with increased expression of antiapoptotic protein Bcl-xL and enhanced DC viability in the PLCH model (37).

Another potential mechanism of DC accumulation in $\mathrm{PLCH}$ is enhanced migration to the lung along well-defined chemokine gradients (14). Circulating immature DCs express the CCR6 receptor that recognizes the ligand CCL20, produced primarily by epithelial cells (16). After pathogenic or environmental activation, DCs undergo maturation and decrease their surface expression of CCR6 and increase their expression of CCR7 (16). CCR7 drives egress of DCs from the lung into mLNs along CCL19 and CCL21 gradients (38). Either an increase in recruitment or a decrease in migra- 
A
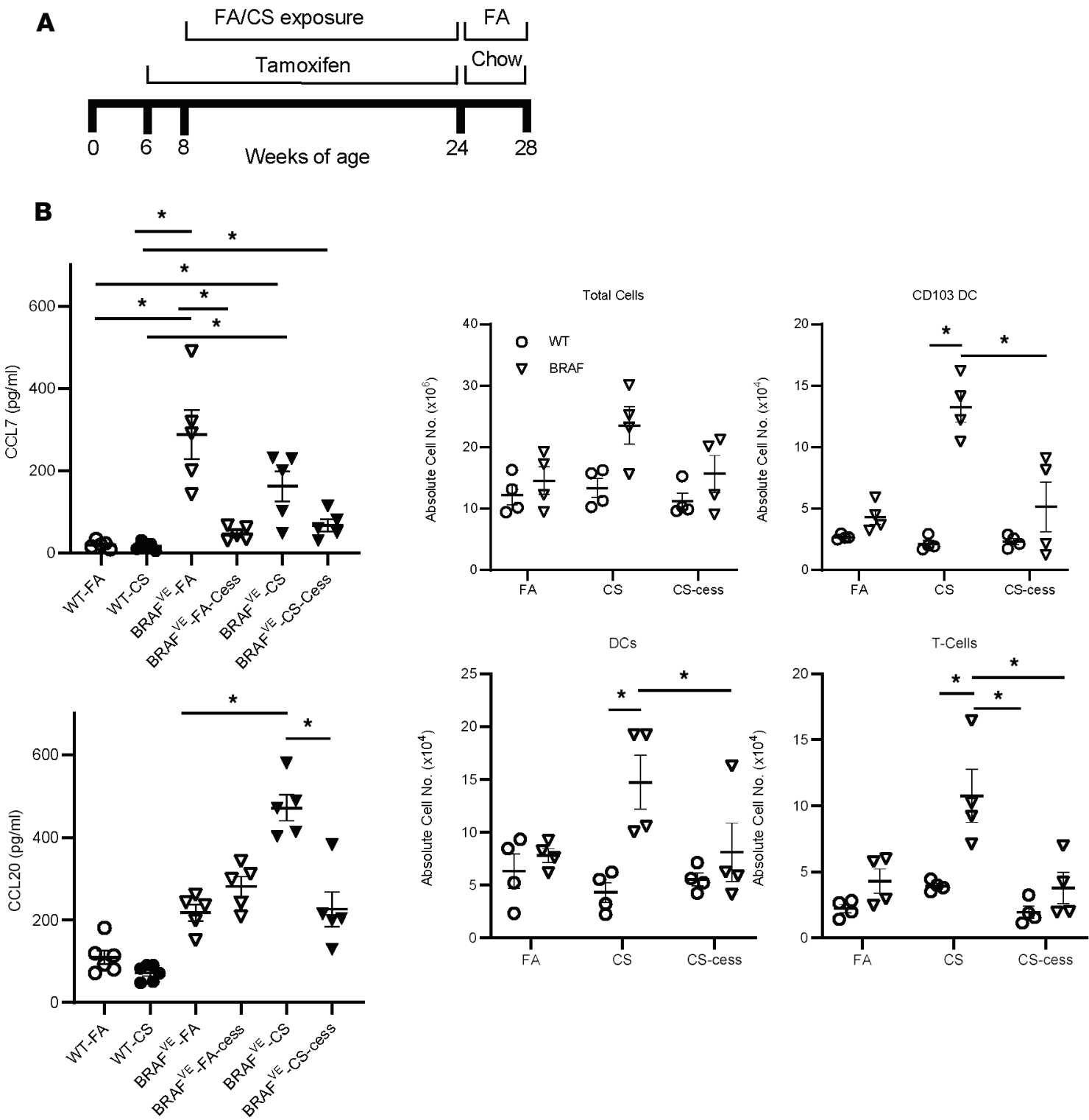

Figure 7. CS and TAM withdrawal ameliorates PLCH phenotypes in BRAFVE mice. (A) Scheme of withdrawal experiments. Mice were treated with TAM chow and exposed to CS and then switched to normal chow and FA for the indicated periods. (B) CCL7 levels in the serum and CCL20 levels in the BAL of mice ( $n=5$ mice/group) at 5 months on TAM chow, after exposure to FA or CS for 4 months, followed by continued treatment or cessation of TAM plus CS cessation (-Cess). (C) The absolute number of inflammatory cells in dissociated lungs of WT or BRAFVE mice ( $n=4 /$ group) exposed to FA or CS for 4 months was determined by flow cytometry. (B and $\mathbf{C}){ }^{*} P<0.05$, 2-way ANOVA with Tukey's multiple-comparisons test. Data shown are mean \pm SEM.

tion to lymph nodes would result in the accumulation of DCs in the lung. Our data reveal increased CCL20 levels in the BAL fluid of BRAFVE mice that further increased after CS exposure. In addition, we showed that BRAFVE DCs had an increased response toward CCR6 ligands but not CCR7 ligands. These findings were confirmed by adoptive transfer experiments that showed that the BRAF ${ }^{\mathrm{VE}} \mathrm{DCs}$ were recruited to the lung more efficiently than WT DCs while the trafficking to the mLNs was unaffected. Collectively, these studies demonstrate that the accumulation of DCs in the lungs of BRAFVE mice is dependent on both increased viability and recruitment. In the lung, pathogenic and environmental exposures regulate the recruitment of DCs from circulation via upregulation of CCL20 secretion (15). Interestingly, we observed intense CCL20 expression in the mononuclear cells of PLCH lesions. This finding, together with the observation that BRAFVE BMDCs exhibited inducible CCL20 expression, indicates that recruited $B R A F^{\mathrm{VE}} \mathrm{DCs}$ are an additional source for CCL20 that may amplify recruitment of circulating pathogenic DCs to the lung. 
The accumulation of BRAF ${ }^{\mathrm{VE}}$ cells in the lung likely represents the initiating event in PLCH pathogenesis. DCs are not the primary cell population in PLCH lesions, and their contribution to tissue destruction and cyst formation is likely limited (39). It is widely believed that cytokines and matrix-modifying enzymes secreted from effector cells, such as macrophages, T cells, and natural killer cells, drive tissue remodeling in PLCH $(40,41)$. We examined mechanisms that mutant DCs might use to regulate accumulation of additional leukocytes to the lung. These studies identified the pleiotropic chemokine CCL7 as a promising candidate based on the BRAF-V600E-dependent expression in DCs. In vivo, we observed increased serum CCL7 in BRAF ${ }^{\mathrm{VE}}$ mice independent of CS exposure. Subsequent studies examining the sources of CCL7 revealed that DCs isolated from the lungs of $B R A F^{\mathrm{VE}}$ mice produced marked amounts of CCL7, which was suppressed with a BRAF-V600E-specific inhibitor. These results were supported using BRAFVE BMDCs. CCL7 is a pleiotropic chemokine that binds to different receptors, including CCR1, CCR2, CCR3, and CCR5, which are found on most leukocytes at varying levels (42). In addition to DCs, many inflammatory cells, including eosinophils, monocytes, macrophages, and T cells, can be recruited in response to CCL7 (43). The role of CCL7 in inflammatory cell recruitment and disease pathogenesis has been studied in many disorders of neutrophilic and allergic lung inflammation $(44,45)$. However, the association between CCL7 and PLCH/LCH had not been reported until a recent study suggested that patients with $\mathrm{LCH}$ with BRAF-V600E mutations, but not other RAS/MAPK mutations, had increased serum CCL7 concentrations (46). Interestingly, increased expression of CCL7 has been reported in other BRAF-V600E-related neoplasms, such as thyroid cancers (47). In addition, inflammatory cytokines, including IL- $1 \beta$ and TNF- $\alpha$, can increase CCL 7 secretion from rat astrocytes through MAPK-dependent pathways, and CCL7 has been shown to promote colon cancer metastasis via the ERK/JNK pathway $(48,49)$. However, the mechanisms by which BRAF-V600E mutations regulate CCL7 are currently unknown. Importantly, the fact that the production of CCL2 and CCL5 was not increased from $\mathrm{BRAF}^{\mathrm{VE}} \mathrm{DCs}$ indicates that the association between BRAF-V600E mutation and CCL7 induction is specific. Together, these studies indicate that the BRAF-V600E-associated increase in CCL7 may represent a mechanism for the DC-mediated recruitment of other inflammatory cells to the PLCH lesions.

The mouse model and limited human data suggest CCL7 may be a potential biomarker in PLCH. A limited analysis revealed nearly undetectable numbers of cells producing CCL7 in controls or patients with PLCH. However, flow analysis revealed that 2 of 3 BRAF-V600E patients produced CCL7 in CD11 $\mathrm{C}^{+}$ PBMCs following activation. The reasons for this discrepancy between the mouse model and patients with $\mathrm{PLCH}$ are possibly differences in the abundance of mutant cells. We suspect that patients with PLCH have a very low abundance of mutant cells whereas the $B R A F^{\mathrm{VE}}$ mice presumably have a higher and more consistent mutation frequency in circulating cells. A recent study examining BRAF-V600E mutations in pediatric $\mathrm{LCH}$ revealed that the proportion of DCs or monocytes carrying the mutations varied between $0.02 \%$ and $25.0 \%$, with the majority of patients having less than $1 \%(10)$. Although pediatric LCH manifests as a severe, systemic, multifocal disease, the spectrum of mutations in the myeloid/monocyte lineages are nearly identical to those of PLCH. It is possible that even lower mutation frequencies that are present in patients with PLCH result in the milder, highly restricted organ involvement patterns observed in that population. In addition, the point in the DC differentiation pathway where the mutation occurs appears to determine the aggressiveness and severity of LCH (10). Future studies examining the frequency and scope of mutations in a large PLCH patient cohort are required to answer these important questions about whether PLCH shares these same pathogenic determinants.

In addition to the molecular and cellular mechanisms controlling the accumulation of DCs and other inflammatory cells, the mechanisms leading to tissue destruction and cyst formation remain a critical gap in our understanding of PLCH pathogenesis. One hypothesis is that the inflammatory cell infiltration and obstruction of small vessels and capillaries adjacent to terminal bronchioles can lead to tissue necrosis and dilation of small airways and alveoli, which can progress to cyst formation $(50,51)$. Alternatively, lung remodeling due to connective tissue degradation and elastolysis by matrix-degrading enzymes, such as matrix metalloproteinases (MMPs), may contribute to cyst formation in PLCH (52-54). Interestingly, there is a report that thyroid cells harboring the BRAF-V600E mutation exhibit increased MMP expression that is associated with increased matrix invasion in vitro (55). Although macrophages are considered the primary effector cell in lung tissue destruction by MMPs, DCs can also elaborate MMPs, raising the possibility that the BRAF-V600E DCs may contribute to tissue remodeling in PLCH. Additionally, cytotoxic lymphocytes present in PLCH lesions express high levels of granzymes that are capable of directed tissue destruction in the lungs of smokers (56). 
We have shown that both the BRAF-V600E mutation and CS exposure are important in the pathogenesis of PLCH. Our data suggest that the PLCH phenotype in BRAF ${ }^{\mathrm{VE}}$ mice lessened after cessation of smoke and TAM administration (halting the production of BRAF ${ }^{\mathrm{VE}}$ mutant DCs). The amelioration of the PLCH phenotype was associated with a decrease of DC-related cytokine and chemokine levels in the BAL fluid, which further validates the causal role of BRAFVE DCs. Since the discovery of mutations in the RAS/MAPK pathway in LCH, multiple studies have shown that BRAF inhibitors have a beneficial effect in patients with $\mathrm{LCH}(57,58)$. However, BRAF inhibition can cause an alternative activation in the MAPK pathway that can lead to significant adverse effects, such as cutaneous squamous cell carcinomas and keratoacanthomas in patients with melanoma (59). Although the experimental strategy used here broadly targets DCs, monocytes, and macrophages at multiple differentiation stages because of the use of the CD11c promoter as the Cre driver, recent studies in pediatric $\mathrm{LCH}$ patients provide convincing evidence that the mutated cells are most consistent with immature myeloid precursors. In this population, although mutations may occur at any point along the myeloid lineage (including monocytes, macrophages, and even B cells), similar effector functions of aberrant migration, invasion, and tissue destruction result, supporting classification of pediatric LCH and other LCH disorders (10) as myelomonocytic neoplasms. Our model identifies a number of potential targets for preclinical testing of new and existing therapeutics and drug combinations targeting the RAS/MAPK pathway, the CCL20 axis, and the CCL7 axis. These studies identify new roles for the BRAF pathway in regulating DC biology, including migration, survival, and effector cell functions. Ultimately, this model will be useful for identifying the cellular and molecular pathways driving the initiation and progression of $\mathrm{PLCH}$ and for developing novel therapies.

\section{Methods}

Study design. The purpose of this study was to establish an animal model of PLCH to study the pathogenesis of this rare lung disease. The primary endpoints were qualitative histologic and IHC assessments and inflammatory cell and cytokine quantification in lavage fluid, lung tissue, and serum of mice exposed to CS for up to 4 months. To decipher the pathogenesis of PLCH on a molecular level, we developed a series of in vitro and ex vivo experiments using BRAF-V600 $\mathrm{E}^{+}$pulmonary $\mathrm{CD} 11 \mathrm{c}^{+}$cells and BMDCs. To determine the reversibility of the PLCH phenotype, we discontinued the CS exposure and TAM treatment of our mouse model. Furthermore, we complemented the in vivo work by applying the BRAF-V600E-specific inhibitor in our in vitro systems. To identify biomarkers that have potential relevance to humans, we measured a panel of cytokines/chemokines in mice and in the serum and PBMCs of patients with PLCH. The BRAF/CCL7 axis was explored using the A-375 melanoma cell line (ATCC). We determined group sizes necessary for adequate statistical power using preliminary data sets. Each experiment was repeated at least 3 times independently with $n$ of 4 or more in each group. There was no randomization used in the experiments, and we did not exclude any samples. The investigators were not blinded to group allocation during collection and analysis of the data. Measurements and counting of lung nodules and cysts were performed by 2 independent blinded lab members.

Mice. C57BL/6J, CD45.1, CD45.2 WT mice, and BRAFV600E $\mathrm{E}^{\mathrm{f} / \mathrm{fl}}$ mice on a C57BL/6 background were obtained from The Jackson Laboratory (Bar Harbor, Maine, USA). CD11c-CreERT mice on a C57BL/ 6 background were a gift from Maries van den Broek (Institute of Experimental Immunology, University of Zurich, Zurich, Switzerland) and have been previously described (22). A detailed schematic of the mouse strains and breeding is shown in Supplemental Figure 1. Inducible CD11c-BRAFV-600E mice were generated by crossing BRAFV600 $\mathrm{E}^{\mathrm{f} / \mathrm{fl}}$ mice with CD11c-CreERT mice. All experimental groups used mice with 1 BRAFV600E $E^{\mathrm{fl}}$ allele and $1 \mathrm{CD} 11 \mathrm{c}-\mathrm{CreERT}$ allele. Littermates that were negative for the Cre recombinase transgene construct were used as controls. Insertion of the BRAF-V600E allele was accomplished with a diet containing TAM ( $80 \mathrm{mg} / \mathrm{kg}$, Envigo) 2 weeks after weaning. Both male and female mice were included in the study. All mice were housed in the University of Cincinnati animal care facilities.

CS exposure. Mice were exposed to either FA or CS generated from 3R4F Kentucky Reference Cigarettes as previously described (60). Whole-body exposures were performed 4 hours per day, 5 days per week, for up to 4 months with a TE-10z smoking machine attached to an exposure chamber (Teague Enterprises). The concentration of the smoke/air mixture was maintained at $150 \pm 15 \mathrm{mg} / \mathrm{m}^{3}$ total suspended particulates.

Cell isolation. Single-cell suspensions of $\mathrm{mLNs}$ were obtained by passing the nodes through a $100-\mu \mathrm{m}$ nylon mesh. To obtain lung DCs, the lung vasculature was perfused with $10 \mathrm{~mL}$ phosphate-buffered saline (PBS) containing $0.6 \mathrm{mM}$ EDTA, via the right atrium. Lung single-cell suspensions were obtained by using 
a gentle-MACS Dissociator (Miltenyi Biotec Inc) after incubating harvested lung tissues with $100 \mathrm{mg} / \mathrm{mL}$ collagenase type IV and 20,000 U/mL DNase (MilliporeSigma) as described previously (61). After ammonium chloride red blood cell lysis, CD11 $\mathrm{c}^{+}$cells were isolated by positive selection using CD11c magnetic MicroBeads following the manufacturer's protocol (Miltenyi Biotec Inc).

Generation and transfection of BMDCs. BMDCs were purified as previously described (62). Briefly, bone marrow was collected from both femurs, red blood cells were lysed, and the remaining cells were passed through a $100-\mu \mathrm{m}$ cell strainer to remove small pieces of bone and debris. Cells were then washed twice with $\mathrm{Ca} / \mathrm{Mg}$-free PBS and seeded into 10 -cm Petri dishes at $5 \times 10^{5}$ cells $/ \mathrm{mL}$ in complete DC medium (RPMI 1640 containing 10\% FBS, $2 \mathrm{mM}$ L-glutamine, $1 \mathrm{mM}$ sodium pyruvate, $0.1 \mathrm{mM}$ nonessential amino acids, $50 \mu \mathrm{M} \beta$-mercaptoethanol, $1000 \mathrm{U} / \mathrm{mL}$ pen/strep, and $20 \mathrm{ng} / \mathrm{mL}$ GM-CSF). At day 3 , cell culture medium was replaced with fresh complete DC medium. At day 5, nonadherent and loosely adherent cells were collected and reseeded into a 6-well plate at $5 \times 10^{5}$ cells $/ \mathrm{mL}$ in fresh complete DC medium. Ad-Cre and control null virus (SignaGen) were added to the cultures at an MOI of 150. At day 7, nonadherent and loosely adherent cells were collected and used to perform experiments.

Lung lavage, fixation, and pathology. BAL fluid for cytokine analysis and cell enumeration was collected by instillation of $1.0 \mathrm{~mL}$ of buffer A (5 mM EDTA, Ca/Mg-free PBS) into the lungs, followed by aspiration via a tracheal cannula. For pathology, mouse lungs were inflation fixed with buffered formalin and embedded in paraffin for histological analysis as previously described (63). Processing and staining of tissue sections was performed at Cincinnati Children's Hospital Research Pathology Core. Three noncontiguous tissue sections were stained with H\&E, CCL20 (1:50; PA5-68465, rabbit anti-human, Invitrogen, Thermo Fisher Scientific), CD68 (1:25; ab125212, rabbit polyclonal, Abcam), B220 (1:1000; ab10558, rabbit polyclonal, Abcam), and CD3 (790-4341, clone 2GV6, Roche). Cyst-like lesions were identified as tissue clearings greater than $200 \mu \mathrm{M}$ in diameter. Nodules and cyst-like lesions were quantified by 2 blinded reviewers. To estimate the volume of each nodule, we assumed approximate spherical shape and calculated volume $=4 / 3 \pi r^{3}$. Healthy human control and PLCH lung tissues obtained by biopsy were preserved in formalin and embedded in paraffin. Human lung tissue was obtained from the Department of Pathology and Laboratory Medicine at the University of Cincinnati Medical Center.

Flow cytometry. For cell surface marker analysis, cells were blocked with anti-CD16/CD32 (553141, Thermo Fisher Scientific) for 30 minutes followed by incubation with specific monoclonal antibodies at $4^{\circ} \mathrm{C}$ for 30 minutes in flow buffer (FB) $(1 \times \mathrm{Ca} / \mathrm{Mg}$-free PBS, $0.5 \%$ BSA, $0.1 \%$ sodium azide). Cells were washed twice with FB and immediately analyzed by flow cytometry (Attune, Thermo Fisher Scientific). All antibodies were obtained from eBioscience, Thermo Fisher Scientific (PE-cyanine 7 antimouse CD11c [N418], APC-efluor 780 anti-mouse CD11b [M1/70], APC anti-mouse CD86 [GL1], PE anti-mouse PDCA-1 [ebio129c], anti-p-ERK [T202/Y204 clone MILAN8R]); BD Biosciences (PerCP-Cy 5.5 anti-mouse CD103 [M290]); and Cell Signaling Technology (anti-Bcl-xL [54H6]). For p-ERK and Bcl-xL staining, BMDCs were fixed with 4\% PFA for 30 minutes at room temperature followed by fixation/permeabilization with ice-cold methanol ( $90 \%$ final concentration) for 30 minutes. Cells were then washed twice with FB and stained with anti-p-ERK and anti-Bcl-xL. Data were analyzed using FlowJo software (v10) (FlowJo, LLC) or FCS Express V5 (De Novo Software). Cells were first gated on lymphocytes, and doublets were excluded by gating on single cells (FSC-A vs. FSC-W). CD $11 \mathrm{c}^{+}$cells were then gated, and macrophages were discriminated from DCs based on high cellular autofluorescence. DCs were gated as $\mathrm{CD} 11 \mathrm{c}^{+}, \mathrm{MHC} \mathrm{II}^{+}$, and autofluorescence ${ }^{\mathrm{mid} / \mathrm{low}}$ cells. Subsets of DC and CD86 expression were then further gated from $\mathrm{CD} 11 \mathrm{c}^{+}, \mathrm{MHC} \mathrm{II}^{+}$, and autofluorescence $\mathrm{mid}^{\mathrm{m} / \mathrm{low}}$ DC populations. The compensation matrix was determined by using UltraComp eBeads (eBioscience, Thermo Fisher Scientific). Appropriate fluorescence minus 1 and negative controls were used to set gates for the above populations.

Immunofluorescence staining. Immunofluorescence staining was performed on cytospin preparations by double indirect immunofluorescence method as previously described (64). Briefly, slides were blocked with goat serum and then incubated overnight at $4^{\circ} \mathrm{C}$ with rabbit anti-mouse CCL7 (1:200; Biorbyt; polyclonal). Secondary labeling was performed by 24-hour incubation with goat anti-rabbit antibody (1:500; Thermo Fisher Scientific). Nuclei were counterstained with DAPI (MilliporeSigma; 1:2000). Images were captured using a Zeiss LSM710 inverted confocal microscope at the Live Microscopy Core, Department of Molecular and Cellular Physiology, University of Cincinnati. Negative controls (unstained and secondary only) were included in each experiment. 
Adoptive transfers. BMDCs (>90\% purity) were derived from CD45.2 WT and BRAFV-600E mouse bone marrow as described above. BMDCs were transfected with Ad-Cre at day 5. At day 7, a total of 2 $\times 10^{6}$ BMDCs in $50 \mu \mathrm{L}$ PBS were injected into recipient CD45.1-transgenic mice via tail vein. Two days after injection, the lungs and $\mathrm{mLNs}$ were collected and digested from the recipient mice as described. The donor BMDCs in lungs and mLNs were identified by the surface expression of CD45.2 by flow cytometry.

DC stimulation. Freshly isolated pulmonary DCs and BMDCs were maintained in complete DC medium. TLR ligand activation was assessed using $1 \times 10^{5}$ freshly isolated DCs seeded in $100 \mu \mathrm{L}$ of DC medium in 96-well, round-bottom plates. DCs were rested for 1 hour followed by a preincubation with $20 \mathrm{ng} / \mathrm{mL}$ IFN- $\gamma$ (PeproTech) for 2 hours. DCs were then treated for 16 hours with $1 \mu \mathrm{g} / \mathrm{mL}$ poly(I:C) (InvivoGen) or $1 \mu \mathrm{g} / \mathrm{mL}$ LPS (MilliporeSigma). IL-12 p40 and IL-6 in supernatant were then measured by ELISA (Thermo Fisher Scientific).

cAMP assay. CAMP levels were measured using the AlphaScreen cAMP assay kit (PerkinElmer) per the manufacturer's protocol in 384-well plates (PerkinElmer). Briefly, $5 \mu \mathrm{L}$ of cells at $1 \times 10^{6} / \mathrm{mL}$ and $5 \mu \mathrm{L}$ of acceptor bead solution were added to each well in triplicate. Cells were stimulated with $5 \mu \mathrm{L}$ of forskolin (1 $\left.\times 10^{-5} \mathrm{M}\right)$, and the plate was incubated at $37^{\circ} \mathrm{C}$ for 15 minutes. Chemokines $(5 \mu \mathrm{L})$ were then added to a final concentration of $300 \mathrm{ng} / \mathrm{mL}$. After a further 30 -minute incubation at $37^{\circ} \mathrm{C}, 15 \mu \mathrm{L}$ of donor beads were added to all wells. After 1 hour of incubation, cAMP levels were measured using a fluorescence plate reader with excitation wavelengths at 680/30 and emission wavelengths at 570/100 (Synergy HTX, Biotek).

Cell migration assays. Migration assays were performed using 24-well Transwell cell culture chambers with 5.0 pore size (Corning Glass) as previously described (65). Briefly, $3 \times 10^{5}$ day 7 BMDCs were placed in the upper chamber and allowed to migrate toward lower chambers containing recombinant mouse CCL19 (300 ng/mL), CCL20 (300 ng/mL), or CCL21 (300 ng/mL) in complete medium at $37^{\circ} \mathrm{C}$. After 3 hours, the number of cells in the lower chamber was determined by flow cytometry.

$R N A$ extraction and real-time quantitative PCR. Total RNA was extracted using TRIzol Reagent (Invitrogen, Thermo Fisher Scientific) and RNeasy MiniElute kit (QIAGEN). Reverse transcription was performed using the Quantitect Reverse Transcription kit (QIAGEN), per the manufacturer's instructions. Quantitative PCR with chemokine genes was conducted with the Power SYBR Green Master Mix (Thermo Fisher Scientific) with the following primers from Integrated DNA Technologies, Inc: CCL2 (Mm.PT.58.42151692), CCL5 (Mm.PT.58.43548565), and CCL7 (Mm.PT.58.17719534). RPL32 (forward: 5'-GAAGTTCCTGGTCCACAACG-3'; reverse: 5'-GCGATCTCGGCACAGTAAG-3') was used for data normalization. The quantitative PCR with mutation-specific BRAF primers was conducted using the iTaq Universal Supermix (Bio-Rad). Briefly, the reaction was performed with $500 \mathrm{nM}$ of the mutation-specific BRAF-V600E mRNA primer (forward: 5'-TAGGTGACTTTGGTCTAGCCACGGA-3'; reverse: 5'-GTAGCTGGCCGGTCATCAGTTCG-3') and $250 \mathrm{nM}$ of the 6-carboxyfluorescein-labeled hybridization probe (6-FAM-TCAGACGTGTATGCGTTTGGGATTG-MGB-NFQ) at an annealing temperature of $68^{\circ} \mathrm{C}$ for 45 cycles. Experiments were performed in triplicate and relative fold change was determined by the $\Delta \Delta \mathrm{Ct}$ method.

Statistics. Four to 8 mice were used in each group with at least 3 independent experiments. Ex vivo experiments were performed in duplicate on at least 3 separate occasions. Unless otherwise indicated, data are expressed as mean \pm SEM. Statistical comparisons employed 1- or 2-way ANOVA. To adjust for multiple comparisons, we used Tukey's post hoc corrections. $P<0.05$ was considered statistically significant.

Study approval. All animal studies (protocol 06-07-04-01) were carried out in accordance with the NIH Guide for the Care and Use of Laboratory Animals (National Academies Press, 2011) and were approved by the University of Cincinnati Institutional Animal Care and Use Committee. All clinical investigation was conducted according to Declaration of Helsinki principles. Human PBMC samples were obtained from the University of Cincinnati Translational Pulmonary Science Center, and use was reviewed and approved by the University of Cincinnati Institutional Review Board (approval 2013-8157). Serum samples were collected after obtaining informed consent from patients visiting the National Hospital Organization Kinki-Chuo Chest Medical Center (Osaka, Japan).

\section{Author contributions}

HL, MTB, and FXM analyzed data and wrote the manuscript. NG, DWM, KAWB, AK, and AT designed studies and interpreted data. HL, ARO, JF, ZS, YI, and MH performed the experiments and data analysis. 


\section{Acknowledgments}

The authors would like to thank Hans Christian Probst (Institute for Immunology, University Medical Centre Mainz, Germany) and Maries van den Broek (Institute of Experimental Immunology, University of Zurich, Switzerland) for their gift of the CD11c-CreERT mice. This work was funded by support from the NIH (HL119538 to MTB and U54HL127672 to FXM) and the Veterans Administration (I01BX002347 to MTB). Other support and resources were provided by the Center for Environmental Genetics (NIH/ National Institute of Environmental Health Sciences, P30 ES006096) and the Center for Clinical and Translational Science and Training (NIH, 5UL1TR001425)

Address correspondence to: Michael T. Borchers or Francis X. McCormack, Division of Pulmonary, Critical Care and Sleep Medicine, Department of Internal Medicine, University of Cincinnati College of Medicine, PO Box 45267-0564, Cincinnati, Ohio 45267, USA. Phone: 513.558.4831; michael.borchers@uc.edu, mccormfx@ucmail.uc.edu.

1. Vassallo R, Harari S, Tazi A. Current understanding and management of pulmonary Langerhans cell histiocytosis. Thorax. 2017;72(10):937-945.

2. Allen CE, Merad M, McClain KL. Langerhans-cell histiocytosis. N Engl J Med. 2018;379(9):856-868.

3. Vassallo R, Ryu JH, Schroeder DR, Decker PA, Limper AH. Clinical outcomes of pulmonary Langerhans'-cell histiocytosis in adults. N Engl J Med. 2002;346(7):484-490.

4. Aricò M, et al. Langerhans cell histiocytosis in adults. Report from the International Registry of the Histiocyte Society. Eur $J$ Cancer. 2003;39(16):2341-2348.

5. Habib SB, et al. Recurrence of recipient Langerhans' cell histiocytosis following bilateral lung transplantation. Thorax. 1998;53(4):323-325.

6. Roden AC, et al. BRAF V600E expression in Langerhans cell histiocytosis: clinical and immunohistochemical study on 25 pulmonary and 54 extrapulmonary cases. Am J Surg Pathol. 2014;38(4):548-551.

7. Chakraborty R, et al. Alternative genetic mechanisms of BRAF activation in Langerhans cell histiocytosis. Blood. 2016;128(21):2533-2537.

8. Badalian-Very G, et al. Recurrent BRAF mutations in Langerhans cell histiocytosis. Blood. 2010;116(11):1919-1923.

9. Kamionek M, et al. Mutually exclusive extracellular signal-regulated kinase pathway mutations are present in different stages of multi-focal pulmonary Langerhans cell histiocytosis supporting clonal nature of the disease. Histopathology. 2016;69(3):499-509.

10. Berres ML, et al. BRAF-V600E expression in precursor versus differentiated dendritic cells defines clinically distinct LCH risk groups. J Exp Med. 2014;211(4):669-683.

11. Espinosa AV, Porchia L, Ringel MD. Targeting BRAF in thyroid cancer. Br J Cancer. 2007;96(1):16-20.

12. McCain J. The MAPK (ERK) pathway: investigational combinations for the treatment of BRAF-mutated metastatic melanoma. PT. 2013;38(2):96-108.

13. Cravens PD, Lipsky PE. Dendritic cells, chemokine receptors and autoimmune inflammatory diseases. Immunol Cell Biol. 2002;80(5):497-505.

14. Liu H, et al. Dendritic cell trafficking and function in rare lung diseases. Am J Respir Cell Mol Biol. 2017;57(4):393-402.

15. Ito T, Carson WF, Cavassani KA, Connett JM, Kunkel SL. CCR6 as a mediator of immunity in the lung and gut. Exp Cell Res. 2011;317(5):613-619.

16. Sallusto F, et al. Rapid and coordinated switch in chemokine receptor expression during dendritic cell maturation. Eur J Immunol. 1998;28(9):2760-2769.

17. Worbs T, Hammerschmidt SI, Förster R. Dendritic cell migration in health and disease. Nat Rev Immunol. 2017;17(1):30-48.

18. Lommatzsch M, et al. Acute effects of tobacco smoke on human airway dendritic cells in vivo. Eur Respir J. 2010;35(5):1130-1136.

19. Botelho FM, et al. Cigarette smoke-induced accumulation of lung dendritic cells is interleukin-1 $\alpha$-dependent in mice. Respir Res. 2012;13:81.

20. Demedts IK, et al. Accumulation of dendritic cells and increased CCL20 levels in the airways of patients with chronic obstructive pulmonary disease. Am J Respir Crit Care Med. 2007;175(10):998-1005.

21. Tsoumakidou M, Demedts IK, Brusselle GG, Jeffery PK. Dendritic cells in chronic obstructive pulmonary disease: new players in an old game. Am J Respir Crit Care Med. 2008;177(11):1180-1186.

22. Probst HC, Lagnel J, Kollias G, van den Broek M. Inducible transgenic mice reveal resting dendritic cells as potent inducers of CD8+ T cell tolerance. Immunity. 2003;18(5):713-720.

23. Zaynagetdinov R, et al. Identification of myeloid cell subsets in murine lungs using flow cytometry. Am J Respir Cell Mol Biol. 2013;49(2):180-189.

24. Sung SS, Fu SM, Rose CE, Gaskin F, Ju ST, Beaty SR. A major lung CD103 (alphaE)-beta7 integrin-positive epithelial dendritic cell population expressing Langerin and tight junction proteins. J Immunol. 2006;176(4):2161-2172.

25. Tazi A, Moreau J, Bergeron A, Dominique S, Hance AJ, Soler P. Evidence that Langerhans cells in adult pulmonary Langerhans cell histiocytosis are mature dendritic cells: importance of the cytokine microenvironment. J Immunol. 1999;163(6):3511-3515.

26. Mitsutake N, Knauf JA, Mitsutake S, Mesa C, Zhang L, Fagin JA. Conditional BRAFV600E expression induces DNA synthesis, apoptosis, dedifferentiation, and chromosomal instability in thyroid PCCL3 cells. Cancer Res. 2005;65(6):2465-2473.

27. Michaloglou C, et al. BRAFE600-associated senescence-like cell cycle arrest of human naevi. Nature. 2005;436(7051):720-724.

28. Wajapeyee N, Serra RW, Zhu X, Mahalingam M, Green MR. Oncogenic BRAF induces senescence and apoptosis through pathways mediated by the secreted protein IGFBP7. Cell. 2008;132(3):363-374. 
29. Felouzis V, Hermand P, de Laissardière GT, Combadière C, Deterre P. Comprehensive analysis of chemokine-induced cAMP-inhibitory responses using a real-time luminescent biosensor. Cell Signal. 2016;28(1):120-129.

30. Cheng JW, et al. The role of CXCL12 and CCL7 chemokines in immune regulation, embryonic development, and tissue regeneration. Cytokine. 2014;69(2):277-283.

31. Suri HS, Yi ES, Nowakowski GS, Vassallo R. Pulmonary langerhans cell histiocytosis. Orphanet J Rare Dis. $2012 ; 7: 16$.

32. Bernstrand C, Cederlund K, Ashtröm L, Henter JI. Smoking preceded pulmonary involvement in adults with Langerhans cell histiocytosis diagnosed in childhood. Acta Paediatr. 2000;89(11):1389-1392.

33. Bernstrand C, et al. Pulmonary abnormalities at long-term follow-up of patients with Langerhans cell histiocytosis. Med Pediatr Oncol. 2001;36(4):459-468.

34. Brabencova E, et al. Langerhans cells in Langerhans cell granulomatosis are not actively proliferating cells. Am J Pathol. 1998;152(5):1143-1149.

35. Senechal B, et al. Expansion of regulatory T cells in patients with Langerhans cell histiocytosis. PLoS Med. 2007;4(8):e253.

36. Marchal J, Kambouchner M, Tazi A, Valeyre D, Soler P. Expression of apoptosis-regulatory proteins in lesions of pulmonary Langerhans cell histiocytosis. Histopathology. 2004;45(1):20-28.

37. Hogstad B, et al. RAF/MEK/extracellular signal-related kinase pathway suppresses dendritic cell migration and traps dendritic cells in Langerhans cell histiocytosis lesions. J Exp Med. 2018;215(1):319-336.

38. Ricart BG, John B, Lee D, Hunter CA, Hammer DA. Dendritic cells distinguish individual chemokine signals through CCR7 and CXCR4. J Immunol. 2011;186(1):53-61.

39. Travis WD, et al. Pulmonary Langerhans cell granulomatosis (histiocytosis X). A clinicopathologic study of 48 cases. Am J Surg Pathol. 1993;17(10):971-986.

40. Hayashi T, et al. Immunohistochemical study of matrix metalloproteinases (MMPs) and their tissue inhibitors (TIMPs) in pulmonary lymphangioleiomyomatosis (LAM). Hum Pathol. 1997;28(9):1071-1078.

41. Zyada MM. Expression of matrix metalloproteinase-9 and significance of a macrophage assay in eosinophilic granuloma. Ann Diagn Pathol. 2009;13(6):367-372.

42. Lazennec G, Richmond A. Chemokines and chemokine receptors: new insights into cancer-related inflammation. Trends Mol Med. 2010;16(3):133-144

43. Griffith JW, Sokol CL, Luster AD. Chemokines and chemokine receptors: positioning cells for host defense and immunity. Annu Rev Immunol. 2014;32:659-702.

44. Michalec L, et al. CCL7 and CXCL10 orchestrate oxidative stress-induced neutrophilic lung inflammation. J Immunol. 2002;168(2):846-852.

45. Shin K, Hwang JJ, Kwon BI, Kheradmand F, Corry DB, Lee SH. Leukotriene enhanced allergic lung inflammation through induction of chemokine production. Clin Exp Med. 2015;15(3):233-244.

46. Morimoto A, et al. Inflammatory serum cytokines and chemokines increase associated with the disease extent in pediatric Langerhans cell histiocytosis. Cytokine. 2017;97:73-79.

47. Rusinek D, et al. BRAFV600E-associated gene expression profile: early changes in the transcriptome, based on a transgenic mouse model of papillary thyroid carcinoma. PLoS ONE. 2015;10(12):e0143688.

48. Lee YS, et al. Crosstalk between CCL7 and CCR3 promotes metastasis of colon cancer cells via ERK-JNK signaling pathways. Oncotarget. 2016;7(24):36842-36853

49. Thompson WL, Van Eldik LJ. Inflammatory cytokines stimulate the chemokines CCL2/MCP-1 and CCL7/MCP-3 through NFkB and MAPK dependent pathways in rat astrocytes [corrected]. Brain Res. 2009;1287:47-57.

50. Ohdama S, Akagawa S, Matsubara O, Yoshizawa Y. Primary diffuse alveolar septal amyloidosis with multiple cysts and calcification. Eur Respir J. 1996;9(7):1569-1571.

51. Kambouchner M, Basset F, Marchal J, Uhl JF, Hance AJ, Soler P. Three-dimensional characterization of pathologic lesions in pulmonary langerhans cell histiocytosis. Am J Respir Crit Care Med. 2002;166(11):1483-1490.

52. Colombat M, et al. Pathomechanisms of cyst formation in pulmonary light chain deposition disease. Eur Respir J. 2008;32(5):1399-1403

53. Matsui K, Takeda K, Yu ZX, Travis WD, Moss J, Ferrans VJ. Role for activation of matrix metalloproteinases in the pathogenesis of pulmonary lymphangioleiomyomatosis. Arch Pathol Lab Med. 2000;124(2):267-275.

54. Hayashi T, Rush WL, Travis WD, Liotta LA, Stetler-Stevenson WG, Ferrans VJ. Immunohistochemical study of matrix metalloproteinases and their tissue inhibitors in pulmonary Langerhans' cell granulomatosis. Arch Pathol Lab Med. 1997;121(9):930-937.

55. Mesa C, et al. Conditional activation of RET/PTC3 and BRAFV600E in thyroid cells is associated with gene expression profiles that predict a preferential role of BRAF in extracellular matrix remodeling. Cancer Res. 2006;66(13):6521-6529

56. Fairclough L, Urbanowicz RA, Corne J, Lamb JR. Killer cells in chronic obstructive pulmonary disease. Clin Sci. 2008;114(8):533-541.

57. Váradi Z, et al. Effective BRAF inhibitor vemurafenib therapy in a 2-year-old patient with sequentially diagnosed Langerhans cell histiocytosis and Erdheim-Chester disease. Onco Targets Ther. 2017;10:521-526.

58. Haroche J, et al. Reproducible and sustained efficacy of targeted therapy with vemurafenib in patients with BRAF(V600E)-mutated Erdheim-Chester disease. J Clin Oncol. 2015;33(5):411-418.

59. Mattei PL, Alora-Palli MB, Kraft S, Lawrence DP, Flaherty KT, Kimball AB. Cutaneous effects of BRAF inhibitor therapy: a case series. Ann Oncol. 2013;24(2):530-537.

60. Motz GT, Eppert BL, Wesselkamper SC, Flury JL, Borchers MT. Chronic cigarette smoke exposure generates pathogenic T cells capable of driving COPD-like disease in Rag2-/- mice. Am J Respir Crit Care Med. 2010;181(11):1223-1233

61. Wortham BW, et al. NKG2D mediates NK cell hyperresponsiveness and influenza-induced pathologies in a mouse model of chronic obstructive pulmonary disease. J Immunol. 2012;188(9):4468-4475.

62. Inaba $\mathrm{K}$, et al. Generation of large numbers of dendritic cells from mouse bone marrow cultures supplemented with granulocyte/macrophage colony-stimulating factor. J Exp Med. 1992;176(6):1693-1702.

63. Motz GT, et al. Chronic cigarette smoke exposure primes NK cell activation in a mouse model of chronic obstructive pulmonary disease. J Immunol. 2010;184(8):4460-4469. 
64. Osterburg AR, et al. NK cell activating receptor ligand expression in lymphangioleiomyomatosis is associated with lung function decline. JCI Insight. 2016;1(16):e87270.

65. Borchers MT, et al. In vitro assessment of chemokine receptor-ligand interactions mediating mouse eosinophil migration. $J$ Leukoc Biol. 2002;71(6):1033-1041. 